\title{
Slow-Light Optical Buffers: Capabilities and Fundamental Limitations
}

\author{
Rodney S. Tucker, Fellow, IEEE, Pei-Cheng Ku, and Constance J. Chang-Hasnain, Fellow, IEEE
}

\begin{abstract}
This paper presents an analysis of optical buffers based on slow-light optical delay lines. The focus of this paper is on slow-light delay lines in which the group velocity is reduced using linear processes, including electromagnetically induced transparency (EIT), population oscillations (POs), and microresonator-based photonic-crystal (PC) filters. We also consider slow-light delay lines in which the group velocity is reduced by an adiabatic process of bandwidth compression. A framework is developed for comparing these techniques and identifying fundamental physical limitations of linear slow-light technologies. It is shown that slow-light delay lines have limited capacity and delay-bandwidth product. In principle, the group velocity in slowlight delay lines can be made to approach zero. But very slow group velocity always comes at the cost of very low bandwidth or throughput. In many applications, miniaturization of the delay line is an important consideration. For all delay-line buffers, the minimum physical size of the buffer for a given number of buffered data bits is ultimately limited by the physical size of each stored bit. We show that in slow-light optical buffers, the minimum achievable size of $1 \mathrm{~b}$ is approximately equal to the wavelength of light in the buffer. We also compare the capabilities and limitations of a range of delay-line buffers, investigate the impact of waveguide losses on the buffer capacity, and look at the applicability of slow-light delay lines in a number of applications.
\end{abstract}

Index Terms-Electromagnetically induced transparency (EIT), optical delay lines, optical memories, optical propagation in dispersive media, photonic crystals (PCs), slow light.

\section{INTRODUCTION}

V ARIABLE-DELAY optical delay lines or buffers have emerged as key components for future optical networks and information processing systems. Applications of optical delay lines and buffers range from optical packet switches (OPSs) [1]-[5] to optical delay-line filters [6], [7] and microwave photonic devices such as antenna beam formers using optical delays to achieve the so-called "true time delays" in microwave signals [8]. In OPS applications, buffers are required for synchronization of incoming packets and for collision avoidance on outgoing lightpaths. The most practical buffer

Manuscript received November 20, 2004; revised May 12, 2005. This paper was supported by the Defense Advanced Research Projects Agency Chip, Set, Wavelength Division Multiplexing (DARPA CSWDM) award F30602-02-20096 and by the Australian Research Council.

R. S. Tucker is with the ARC Special Research Center for Ultra-Broadband Information Networks, Department of Electrical and Electronic Engineering, University of Melbourne, Victoria 3010, Australia (e-mail: r.tucker@ unimelb.edu.au).

P.-C. Ku is with the Intel Corporation, Santa Clara, CA 95052 USA.

C. J. Chang-Hasnain is with the Electrical Engineering and Computer Science (EECS) Department, University of California at Berkeley, Berkeley, CA 94720 USA.

Digital Object Identifier 10.1109/JLT.2005.853125 technology for OPS applications is based on fiber delay lines combined with optical switches [5]. This style of buffer has proved satisfactory in limited-scale laboratory-based demonstrations of OPS, but it is bulky and does not scale well to fullsize networks, especially in buffering for collision avoidance.

To highlight the scaling problem in large-scale OPSs, consider an OPS or router with 1000 incoming and outgoing channels, each at a data rate of $40 \mathrm{~Gb} / \mathrm{s}$. In electronic routers, the buffering capacity per port is usually equal to around $250 \mathrm{~ms}$ of delay per port [9]. At the $40-\mathrm{Gb} / \mathrm{s}$ data rate, this corresponds to a buffer capacity of around $10 \mathrm{~Gb}-\mathrm{a}$ figure that is used in present-day state-of-the art electronic routers (see, for example, [10]). If single-wavelength optical fiber delay lines were used in place of electronics for this buffering, then the total length of fiber needed for buffering all ports in the router would be approximately $40 \mathrm{Gm}$, or about 150 times the distance from the earth to the moon! Even if we set aside issues of signal distortion by dispersion and the very significant problem of power consumption by the necessary inline amplifiers, these lengths of fiber are quite unrealistic just on the basis of the physical space required to house the delay lines. There have been recent suggestions that adequate network performance can be achieved with two orders of magnitude less buffering than this [9]. In addition, wavelength-division multiplexing (WDM) of multiple stored bit streams on each delay line could reduce the total length. However, while these measures may help a little, the required buffers would still be impractically large.

At the heart of the buffer scaling problem is the issue of the physical size of a bit of data stored on an optical fiber delay line. At $40 \mathrm{~Gb} / \mathrm{s}, 1 \mathrm{~b}$ occupies approximately $5 \mathrm{~mm}$ of length on the fiber. Compare this with complementary metal-oxidesemiconductor random access memory (CMOS RAM), which can store a single bit in an area of less than $1 \mu \mathrm{m}^{2}$ [11]. The clear message here is that for OPS to become viable and for OPS networking to become competitive with electronic networking, it will be necessary to find an optical buffering technology that provides many orders of magnitude reduction in size, i.e., many orders of magnitude increase in storage density. As pointed out above, optical signal processing and microwave photonic applications generally require shorter fiber lengths. However, in these applications, there is a need for greatly improved miniaturization and increased storage density.

New optimism about the possible development of miniaturized optical delay line buffers has been stimulated by a recent surge of research activity in the area of slow light. This research has renewed speculation that slow light may hold the key to practical optical buffering technologies. Recent results in linear slow-light propagation have included very slow or even stopped 


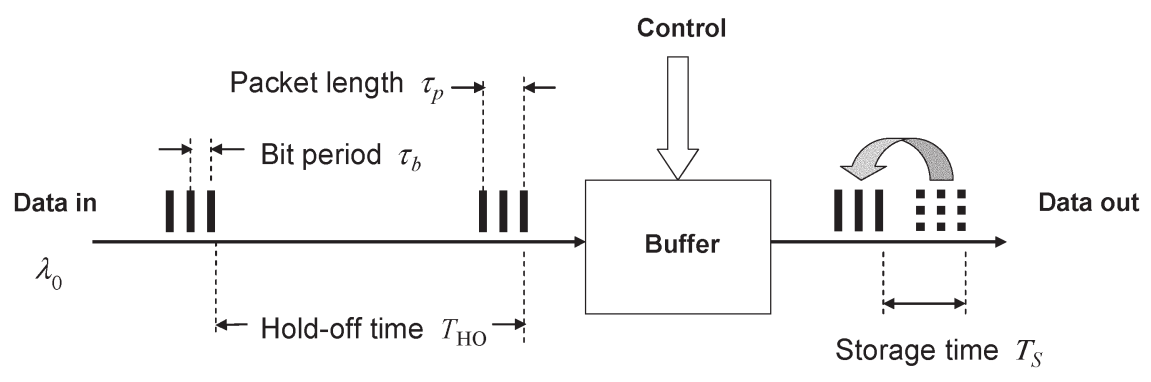

Fig. 1. Optical buffer memory.

light in an atomic vapor using electromagnetically induced transparency (EIT) [12], [13], a slow-down factor of more than 31000 using population oscillation (PO) in quantum-well material [14], EIT in solid-state material [15], and a series of theoretical predictions in coupled resonator optical waveguides [16], and other photonic-crystal (PC)-coupled microcavities [17].

Although these results and proposals appear very promising, it is not yet clear how easily each of these slow-light techniques will translate into practical devices or what engineering tradeoffs need to be made in order to optimize performance. The objective of this paper is to analyze and compare the potential of various slow-light device technologies and to provide a framework for understanding the capabilities of these technologies. It is still "early days" for slow-light optical buffers. There are a number of technical challenges to be overcome before slow-light buffers become fully practical. Indeed, a number of devices and structures that we consider in this paper cannot yet be fabricated with available technology. The focus of this paper is on possibilities and limitations rather than on practical realizations.

We present what we believe to be the first detailed comparison of the capabilities of linear slow-light buffers. The analysis includes a number of different technology approaches to buffering, including EIT, POs in pumped media, and microresonatorbased PC filters. We also consider slow-light delay lines in which the group velocity is reduced by a process of adiabatic bandwidth compression. To provide a baseline reference, these slow-light devices are compared with conventional fiber delay lines. We identify the fundamental physical limitations of a variety of slow-light techniques and propose a number of new "figures of merit" related to storage capacity of optical buffers. These limitations set a maximum achievable delay time-bandwidth product in the device and lead to a lower bound on the achievable density of data storage in a delay line.

For all delay-line buffers, the minimum physical size of the buffer is ultimately limited by the maximum density of storage of data bits in the buffer or, equivalently, by the physical size of each stored bit. It is shown that in slow-light optical buffers, the minimum size of $1 \mathrm{~b}$ is approximately equal to the wavelength of light in the buffer. It is shown that for ideal delay lines, the maximum achievable storage density is approximately $1 \mathrm{~b}$ per unit wavelength. EIT/PO devices can, in principle, approach this ideal storage density, but only for very short devices. It is also shown that the loss-limited capacity of a delay line is a useful measure of the practical limit on capacity. The paper concludes that slow-light buffers appear unlikely to provide a practical solution to contention resolution in OPSs, where very large data capacity is required. However, it is expected that slow-light buffers will find applications where compact all-optical buffers are needed, with capacities of around $1 \mathrm{Mb}$ or less.

The paper is structured as follows. Section II defines some of the key parameters of delay-line optical buffers and considers some basic properties of slow-light delay lines. Section III concentrates on a class of slow-light buffers that have been the focus of much of the recently published experimental work on slow light. These buffers (called Class A buffers) use techniques such as EIT, PO, or PC filters to produce a controllable low group velocity. Section III considers the capabilities and limitations of Class A. Section IV is devoted to the analysis of a second class of buffer (called Class B buffers) in which the bandwidth and the group velocity of the stored data are controlled. Section V considers devices that comprise a mix of the above classes of device, and Section VI looks at a number of applications of slow-light buffers and provides a comparison of the capabilities and limitations of a number of buffers. Section VII summarizes the main conclusions of the paper.

\section{OpticAl BUfFER MEMORY USING Slow-Light Delay LineS}

Fig. 1 shows the layout of a generic optical buffer memory. This generic description applies not only to slow-light optical buffers but also to other buffers such as fiber delay-line buffers. Incoming optical data enter from the left and stored data exit the buffer to the right. For simplicity, we begin by considering input and output signals at a single optical wavelength $\lambda_{0}$. Section III-E generalizes the analysis to include multiple wavelength-multiplexed channels. The buffer is controlled via one or more control inputs. These control signals are represented by an arrow in Fig. 1. In general, the control signals can specify a number of operations, including the physical location of the stored data in the buffer and the storage time.

One kind of memory, with applications in OPS, is the firstin-first-out (FIFO) memory. In general, a FIFO memory is a read-write device that is sequentially accessible and in which the data rate of the input and output can differ [11] while keeping the same sequence of data at the output and input. Optical delay-line buffers are inherently FIFO devices, but optical FIFO memory generally operates with the same input and output data rates. In some applications, such as optical signal processing and in some packet-switching architectures, it may be desirable for the optical buffer to behave like a RAM, in which individual bits or groups of bits of data can be stored and retrieved at will. True RAM operation is difficult 
to achieve in optical buffers. However, limited RAM operation may be achievable with integrated-optical slow-light optical buffer technology (see Fig. 3).

Fig. 1 schematically shows some incoming and outgoing optical data and defines a number of key characteristics of the data and the buffer. The incoming data comprise a series of packets (i.e., strings of bits) centered at the optical radian frequency $\omega_{0}$. The free-space wavelength corresponding to $\omega_{0}$ is $\lambda_{0}$. The length of the packets is $\tau_{p}$, the bit period of the data in each packet is $\tau_{b}$, and the baseband bandwidth of the input data is $B_{\text {packet }} \cong 1 / \tau_{b}$, assuming a return-to-zero (RZ) format. The optical bandwidth of the data is $\Delta v=\Delta \omega / 2 \pi \cong 2 B_{\text {packet }}$. The number of bits in each packet is $b_{\text {packet }}=\tau_{p} / \tau_{b}$.

Two important parameters that characterize the overall performance of the buffer are the storage time $T_{S}$ and the hold-off time $T_{\mathrm{HO}}$. In Fig. 1, the output packet shown with dashed lines represents the input data as it would have emerged from the buffer if it passed directly through the buffer without any controlled delay. The storage time is the delay between this and the buffered packet. In Fig. 1, the storage time $T_{S}$ is shown as being larger than the packet length $\tau_{p}$, but $T_{S}$ can be smaller than $\tau_{p}$. The hold-off time is a "dead time" after data have been loaded into the buffer. During the hold-off time, the buffer cannot accept any additional data. This limits the rate at which packets can be loaded into the buffer. We define the hold-off time to be the minimum allowable time between the leading edge of an incoming packet or group of packets that have been stored in the buffer and the leading edge of the following packet or group of packets that are to be stored independently of the first group.

Another important parameter is the storage time-bandwidth product (or the delay-bandwidth product) $T_{S} B_{\text {packet }}$. We will show in Section III that in Class A delay lines, the delaybandwidth product is equal to $T_{S} B_{\text {packet }}=T_{S} / \tau_{b}=C$, where $C$ is the capacity of the buffer (i.e., the maximum number of bits that can be stored in the buffer).

Since the optical buffers considered here are analog (i.e., nondigital) devices, their performance is ultimately limited by optical loss. In this paper, we characterize the optical loss in terms of the field attenuation constant $\alpha_{R}$ (with units of nepers per meter) and the length $L$ of the buffer. To characterize the capacity of an optical buffer with optical loss, we introduce the concept of the delay-bandwidth product per neper of loss. The delay-bandwidth product per neper of loss is the delay-bandwidth product when the delay line has a length of $1 / \alpha_{R}$. It represents the capacity of a buffer with a total loss of $1 \mathrm{~Np}(8.7 \mathrm{~dB})$.

It is important to recognize that the storage time $T_{S}$ defined above refers to a change in propagation time of the data through the buffer in response to a change in the control signal(s) that determines the buffer's operation. Without a controllable change in propagation time, the buffer is just a fixed delay that, by itself, does not constitute a buffer. In some types of optical buffer, $T_{S}$ is quantized. In others, it can take a large or infinite number of values over some range. Note that the definitions above specifically refer to data in the form of packets. However, our analysis also applies to buffers that store only single bits of data rather than packets. For the special case of single-bit data, we simply replace $\tau_{p}$ by $\tau_{b}$ in the analysis.
There are a number of additional parameters that are important in characterizing the properties of optical buffers. These include the physical dimensions of the device, signal distortion through dispersion and other nonideal filtering characteristics, and noise. Finally, the power dissipation per stored bit is a fundamentally important parameter for all memories, whether optical or electronic. In later sections of this paper, we consider the physical length of buffers and compare the storage density offered by different buffering technologies. This paper aims to explore the fundamental limitations of performance in terms of storage time and storage density. Considerations of, signal distortion caused by dispersion, and power requirements, are beyond the scope of this paper.

Practical optical buffers can be constructed using a number of basic building blocks, three of which are shown in Fig. 2 . The building blocks can be used either in isolation or combined to increase capacity, flexibility, and the range over which the storage time $T_{S}$ can be changed. Fig. 2(a) represents a very basic buffer, comprising a delay line with a controllable delay. Examples of this kind of device include controllable slow-light devices and mechanically adjustable delay lines. As pointed out above, simple (noncontrollable) fiber delay lines are not buffers. Fig. 2(b) shows a delay line connected to a cross-point switch in a delay-loop configuration. When the cross-point is in the bar state, the cross-point switch directs incoming data directly to the output and reinjects data emerging from the delay line back into the delay line. When the cross-point is in the cross state, it directs incoming data into the delay line and routes data from the output of the delay line to the output of the buffer.

Unlike the buffer in Fig. 2(a), the delay line in the buffer in Fig. 2(b) can be either fixed or controllable. Thus, the cross-point enables noncontrollable fiber delay lines to be used in buffers. If the delay line has a controllable delay, the buffer provides a larger range of storage times. Demonstrations of OPS using delay-loop buffers with fiber delay line date back to the origins of OPS [5]. Delay-loop buffers have the advantage that longer delays can be achieved than could otherwise be achieved with the same length of fiber. In addition, an array of parallel delay-loop buffers (see Fig. 3) can provide RAM-like capability. However, the number of bits stored in the loop buffer is no greater than in a controllable delay line of the same length. Fig. 2(c) shows a third basic buffer building block that has two inputs and two outputs. Two cross-points direct the incoming data directly either to the output or to the output via the delay line. Cascades of this building block, using fiber delay lines with a range of different fixed delay times, have been proposed for OPS applications. Details of this building block are given in [18].

Fig. 3 shows how the number $M$ of buffers $B 1-B M$ can be combined either in cascade [Fig. 3(a)] or in parallel [Fig. 3(b)]. A FIFO buffer can be constructed using the cascade structure in Fig. 3(a) by combining variable delay lines. A RAM buffer can be constructed using the parallel structure in Fig. 3(b) with parallel FIFO buffers or delay-loop buffers combined using switches at the input and output.

The basic variable delay line in Fig. 2(a) is a fundamentally important element for all optical buffers, including those with and without cross-points. In Sections III and IV, we 
(a)

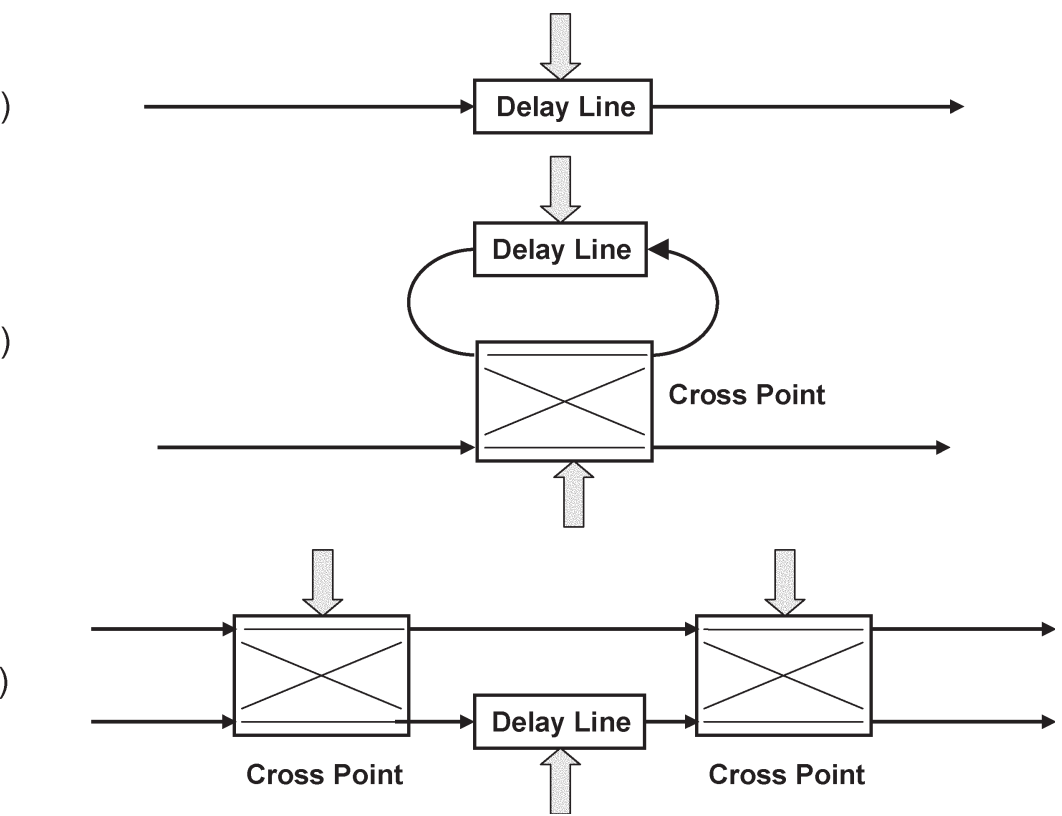

(b)

Fig. 2. Unit optical buffers. (a) Variable delay line. (b) Delay loop. (c) Staggered delay line. Thin lines represent optical paths. Broad arrows represent control signals.

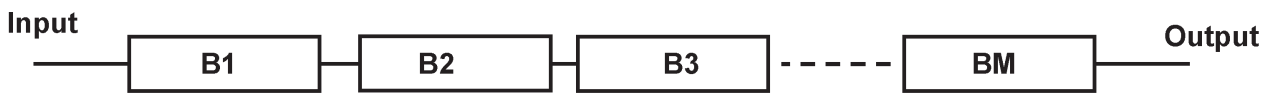

(a)

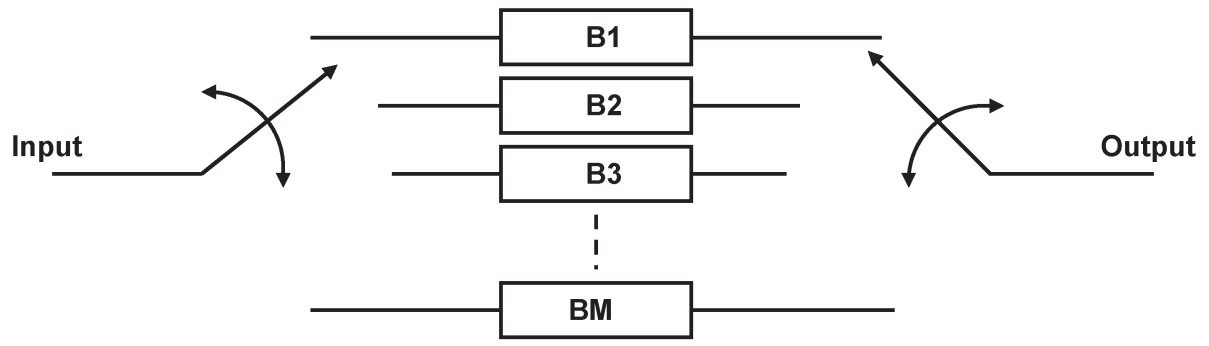

(b)

Fig. 3. (a) Cascaded and (b) parallel buffers.

analyze the propagation properties of variable delay lines using simple models of the devices. Fig. 4 defines some parameters used in the analysis. The length of the delay line is $L$, and $x$ represents the distance of a data bit or packet from the input of the delay line. Fig. 4 shows a packet in which the number of bits in the packet $b_{\text {packet }}$ is equal to five. The physical spacing of each bit is $L_{\mathrm{bit}}$. The capacity $C$ of the delay line (i.e., the maximum number of bits that could be stored on the delay line) is $C=T_{S} B_{\text {packet }}=L / L_{\mathrm{bit}}$. Note that in some applications, it may be desirable to operate the delay line at less than full capacity, as is the case in Fig. 4. In Section I of this paper, we highlighted the importance of miniaturization in the design of optical buffers. To achieve a small buffer, it is necessary that the physical size or spacing $L_{\mathrm{bit}}$ of the stored bits is small. Another way of looking at this is in terms of the storage density in the device, i.e., the number of stored bits per unit length of the buffer. For a delay line of length $L$, as shown in Fig. 4, the storage density SD of the device is $\mathrm{SD}=C / L=L_{\mathrm{bit}}^{-1}$ and the size $L_{\mathrm{bit}}$ of a stored bit is $L_{\mathrm{bit}}=$
$L /\left(T_{S} B_{\text {packet }}\right)$. In the following sections, we will examine the limitations on SD and $L_{\mathrm{bit}}$ for a number of device types.

The transmission characteristics of the delay line in Fig. 4 are controlled by the vector of control signals $\zeta(t)$. The control signals (or control signal in some cases) determine the propagation characteristics along the delay line. We model the delay line in terms of its residual loss $\alpha_{R}$ (in nepers per meter) and its group velocity $v_{g}$. We assume that the loss is constant, but in general the group velocity is a function both of position $x$ and time $t$. Thus, we can write the group velocity $v_{g}$ as

$$
v_{g}(x, t)=\frac{\mathrm{d} x}{\mathrm{~d} t}
$$

where the group-velocity profile $v_{g}(x, t)$ is controlled by the input $\zeta(t)$. In EIT slow-light devices, for example, the group velocity $v_{g}$ can be changed as a function of time by controlling the wavelength and power of the pump laser [19], [20]. Similarly, the profile of $v_{g}$ can be controlled as a function of 


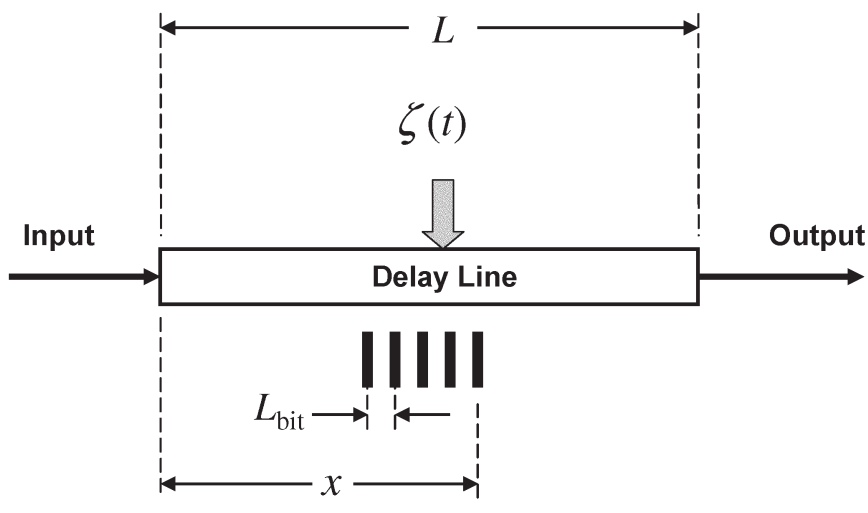

Fig. 4. Variable delay line.

position $x$ by appropriate positioning of the pump beam. In the present paper, we assume that $v_{g}$ can be separated into a product of two terms, one dependent on time and one dependent on position. Thus, we put

$$
v_{g}(x, t)=v_{x}(x) v_{t}(t)
$$

where $v_{x}(x)$ is a function of position only and $v_{t}(t)$ is a function of time only. Section III considers delay lines in which $v_{t}(t)$ is a constant (Class A delay lines), and Section IV considers delay lines in which $v_{t}(t)$ is not constant (Class B delay lines).

\section{BufFers With GROUP Velocity INDEPENDENT OF TIME (CLASS A)}

This section examines variable delay lines in which the group velocity remains constant while data are stored in the delay line. An example of an application for variable delay lines of this type is given in Fig. 5, which shows how a cascade of $M$ variable delay lines [see Fig. 3(a)] can function as a FIFO buffer. For simplicity, in this example, the group velocity in each of the $M$ delay lines can be set at one of two values $v_{g 1}$ or $v_{g 2}$ using the control signals $\zeta_{1}(t)-\zeta_{M}(t)$. Light at $v_{g 1}$ is "full speed," and light at $v_{g 2}$ is at "low speed," i.e., slow light. The FIFO buffer operates as follows. The group velocity in the first delay line (DL1 in Fig. 5) is always equal to $v_{g 2}$. If a packet arrives at the input of the first delay line while another packet is stored in DL1, then the incoming packet is not accepted. If there is no packet currently stored in DL1, the incoming packet is loaded into DL1. The group velocity is $v_{g 2}$ in all delay lines between the most recently accepted packet and the packet closest to the output. Delay lines to the right of the packet closest to the output also have group velocities $v_{g 2}$. When it is required to read out the packet closest to the output, the group velocity in all delay lines to the right of that packet is set to group velocity $v_{g 1}$. As a concrete example, Fig. 5 shows a snapshot of a number of 3-b packets stored in the buffer. The buffer closest to the output is currently in DLM-1, and it has been decided to read out this packet to the output. To do this, the group velocity in DLM has been set to $v_{g 1}$.

In each of the $M$ delay lines in the FIFO buffer in Fig. 5, the group velocity remains at a fixed value while a packet is present in the delay line. The group velocities in the delay lines are adjusted to new values only when there is no packet in the line. We denote delay lines in which the group velocity remains constant as "Class A." Note that even though the group-velocity profile does not change while data are stored, Class A delay lines are variable. To illustrate in more detail the characteristics of this kind of delay line, Fig. 6 schematically shows a train of periodic pulses in a Class A slow-light delay line. Pulses enter from the right; in region 1 , the group velocity is $v_{g 1}$, and at position $x_{A}$ the pulses enter a slow-light delay region with low group velocity $v_{g 2}$ (region 2 ). The pulses are reaccelerated to $v_{g 1}$ when they enter region 3 at position $x_{B}$.

The group-velocity distribution along the device length is shown in Fig. 6(a) and (b) as a "snapshot" of the pulses in the device. Note that the pulsewidth and the spacing decrease with the group velocity. In some devices such as PC-based waveguides, the field intensity of the pulses increases in the slowlight region. This increase in the field intensity of slow light can be used to enhance nonlinear material effects [17]. As shown in Fig. 6, it is possible to buffer data in a Class A delay line even if the packet length $\tau_{p}$ is larger than the storage time $T_{S}$. In the situation depicted in Fig. 6, the number of bits stored (i.e., the capacity) $C<b_{\text {packet}}$. On the other hand, if the packet length

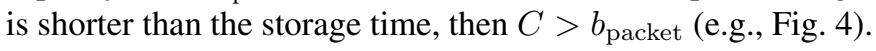

In Fig. 6, the transitions in group velocity from "high" to "low" and "low" to "high" are abrupt. In Fig. 7, we show a snapshot of pulses in transition across a graded transition in group velocity. The group velocity decreases linearly as a function of $x$ through the transition region from a value of $v_{g 1}$ at position $x_{A 1}$ to a value of $v_{g 2}$ at position $x_{A 2}$. Fig. 7 shows how the pulses change as they slow down. Because of the slope of the $v_{g}$ characteristic, there is a group-velocity differential across the pulses as they move across the transition region. We represent this group-velocity differential by $\Delta v_{g}$ as shown in Fig. 7. The group-velocity differential is given by

$$
\Delta v_{g}=\frac{v_{g 1}-v_{g 2}}{x_{A 2}-x_{A 1}} L_{b}(x)
$$

where $L_{b}(x)$ is the pulsewidth at position $x$. It is easy to show that this group-velocity differential causes the pulses to compress in space as they cross the transition region. The physical length $L_{b 2}$ of a pulse when it reaches the slow-light region is given by

$$
L_{b 2}=\frac{L_{b 1}}{S}
$$

where $L_{b 1}$ is the physical length of the pulse in region 1 and $S=v_{g 1} / v_{g 2}$ is the slow-down factor [19].

Note that for Class A buffers, even though the pulses compress in space, they do not compress in time. This is because the reduction in pulsewidth is matched by a corresponding reduction in group delay. Thus, the bit period $\tau_{b}$ and the bandwidth of the data remain unchanged through the device length. This is true for any shape transition characteristic in Class A devices and not just the linear characteristics shown in Fig. 7. (We show in Section IV that the situation is very different for Class B devices.) It is worth repeating here that in Class A, delay lines are defined as delay lines in which the group velocity does not 


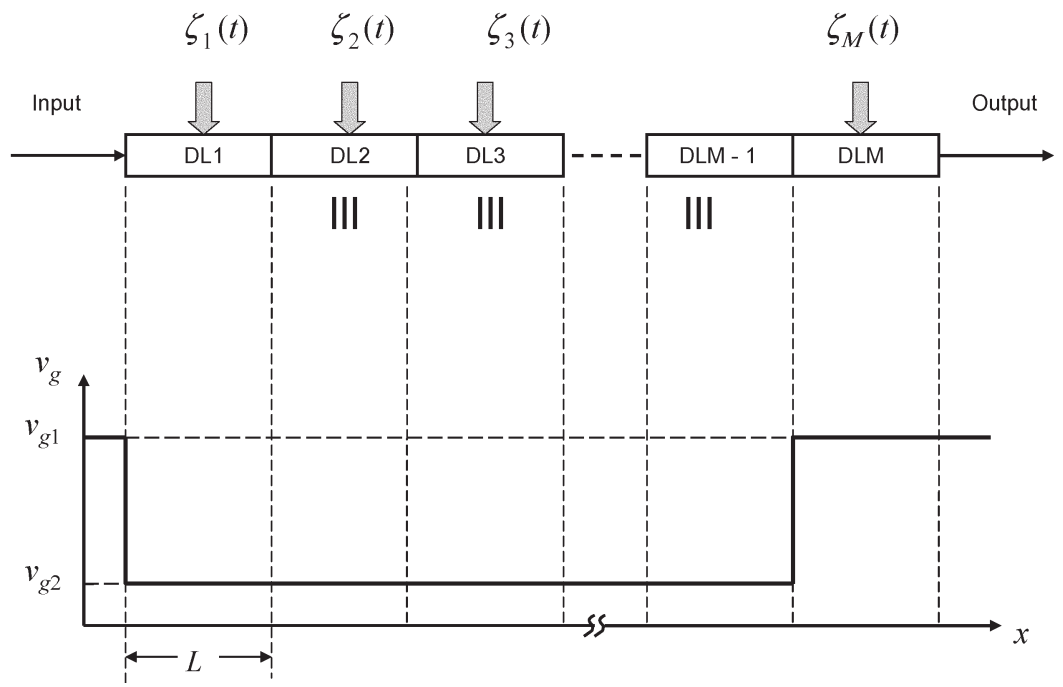

Fig. 5. FIFO buffer using cascaded Class A delay lines.

(a)

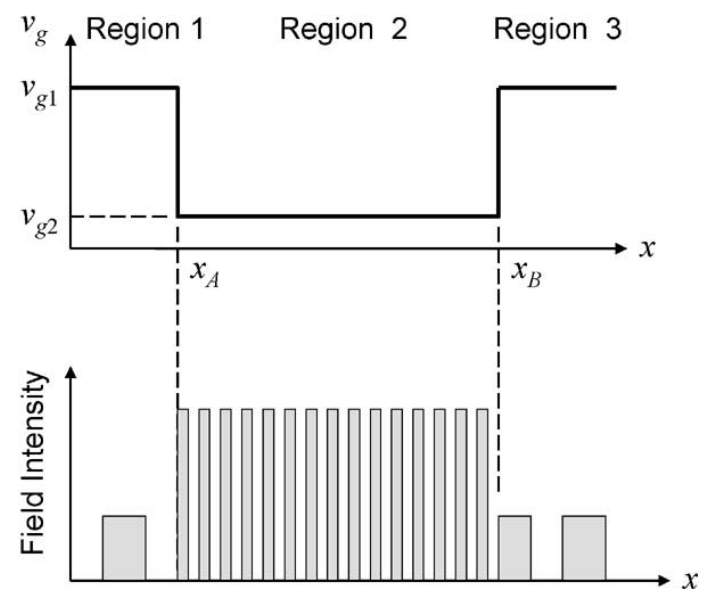

Fig. 6. Pulses in Class A slow-light variable delay line.

change when data are in the delay line. However, this does not preclude the delay from changing when no data are present. Indeed, as pointed out earlier, a delay line whose delay cannot be changed is not an optical buffer. In Class A delay lines, the delay through the device can be changed through changes to the group velocity $v_{g 2}$ in the slow-light region and/or through changes to the length of the slow-light region (i.e., via changes to $x_{A}$ and $x_{B}$ in Fig. 6).

We now look in detail at how the group velocity of Class A devices can be controlled by adjusting the refractive-index profile. Recall that the key objective of all delay lines considered here is to provide low group velocity and to enable control over this low group velocity. We begin the analysis by considering the optical field $E(t, x)$ at position $x$ of a wave of optical frequency $\omega$ propagating in a waveguide or other medium

$$
E(t, x)=E_{o} e^{j(\omega t-k x)}
$$

where $k$ is the wave vector given by $k^{2}=\omega^{2} \varepsilon / c^{2}$, $\varepsilon$ is the dielectric constant given by $\sqrt{\varepsilon(\omega, k)}=n(\omega, k)+j c \alpha / 2 \omega$, $n(\omega, k)$ is the refractive index, $\alpha$ is the attenuation constant, $x$ is the position on the waveguide, $c$ is the free-space velocity of light, and $E_{o}$ is a constant. This paper focuses on slow light in- (a)

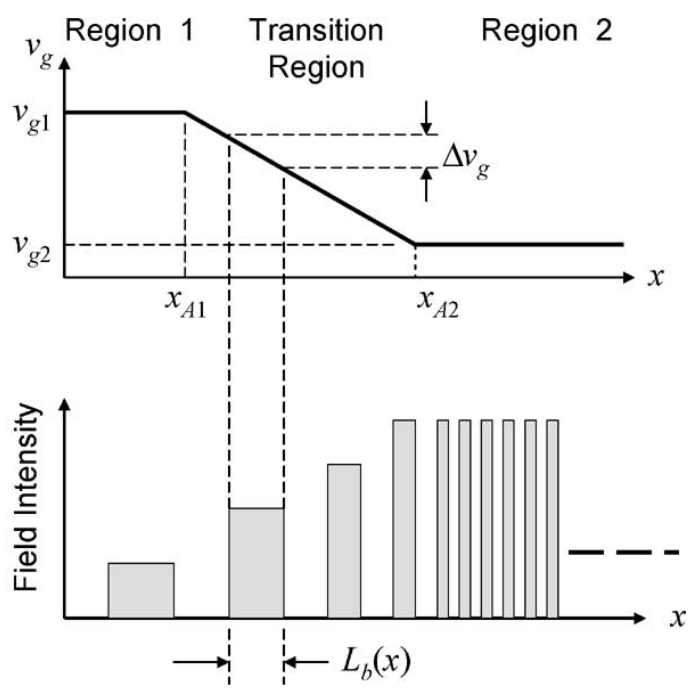

Fig. 7. Pulses in Class A slow-light variable delay line with transition region.

duced by material effects and slow light produced by devices in which a wave at frequency $\omega$ propagating with a wave vector $k$ "feels" that it is traveling in a homogeneous medium with an effective refractive index $n(\omega, k)$ even if the medium has a nonuniform index distribution $n(x, y, z)$ such as a Bragg grating or PC filter.

The group velocity $v_{g i}$ in waveguide $i$ is defined in terms of the waveguide propagation constant $k_{i}$

$$
v_{g i}=\frac{\partial \omega}{\partial k_{i}}=\frac{c}{n_{i}+\omega \frac{d n_{i}}{d \omega}}
$$

where $c$ is the velocity of light and $n_{i}$ is the effective refractive index in waveguide $i$. Thus, the slow-down factor when a signal passes across the boundary between two waveguides (waveguide 1 and waveguide 2) is

$$
S(\omega)=\frac{v_{g 1}}{v_{g 2}}=\frac{n_{2}+\omega \frac{d n_{2}}{d \omega}}{n_{1}+\omega \frac{d n_{1}}{d \omega}}=\frac{n_{g 2}}{n_{g 1}}
$$



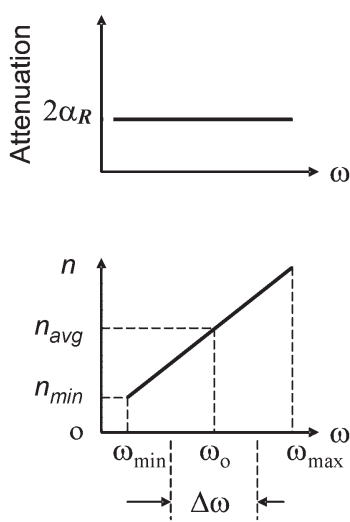

(a)

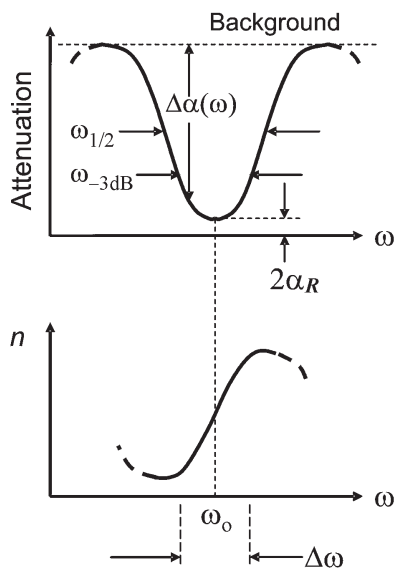

(b)
Fig. 8. Characteristics of slow-light delay lines.

where $n_{g i}=n_{i}+\omega\left(d n_{i} / d \omega\right)$ is the group index in waveguide $i$. It is easy to see from (7) that the slow-down factor can made large by ensuring that the $d n_{2} / d \omega$ term is larger than the $d n_{1} / d \omega$ term. In many of the slow-light devices, we consider here, waveguide 2 is designed to produce slow light, while waveguide 1 is not a slow-light waveguide (i.e., $d n_{1} / d \omega \approx 0$ ). Thus, the slow-down factor in (7) is controlled primarily by the $\omega\left(d n_{2} / d \omega\right)$ term. For low-distortion slow-light propagation, the term $\omega\left(d n_{2} / d \omega\right)$ in (7) should be large and independent of the frequency $\omega$ across the signal passband. In most practical situations, the fractional bandwidth of the signal is small, and this requirement translates into a requirement that the effective refractive-index slope $d n_{2} / d \omega$ is large and independent of frequency across the passband, i.e., that a plot of $n_{2}$ versus $\omega$ is a straight line with large slope.

We now address the question "What is the best refractive index and attenuation characteristic that could be devised to maximize the slow-down factor and minimize distortion by frequency-dependent attenuation and dispersion?" The answer is that the slope of the effective refractive-index versus frequency plot should be maximized across the signal bandwidth and the attenuation should be independent of frequency. Fig. 8(a) shows this ideal attenuation and effective refractive index. The attenuation is constant, with a value of $\alpha_{R}$, and the refractive index is a linear function of frequency and has its minimum value $n_{\min }$ at optical frequency $\omega_{\min }$ and its maximum value at optical frequency $\omega_{\max }$. The average effective refractive index across the signal band is $n_{\text {avg }}$, and the center frequency $\omega_{0}$ of the signal is aligned with the center of the region of linear slope. To avoid signal distortion, the bandwidth of the optical signal $\Delta \omega$ must satisfy the condition $\Delta \omega \leq$ $\left(\omega_{\max }-\omega_{\min }\right)$. In other words, an ideal delay line has an allpass characteristic [24] across the full signal bandwidth.

Fig. 8(b) shows the attenuation and real refractive index $n$ of a practical (nonideal) delay line based on EIT [19]. The same model also applies to delay lines based on PO in quantum-well material [14]. The ideal characteristic in Fig. 8(a) has an attenuation that is flat from $\omega_{1}$ to $\omega_{2}$. In contrast, the EIT characteristic in Fig. 8(b) has an absorption in which there is a "hole" in the absorption characteristic of the transmission medium in a wave- length region of high attenuation. The minimum attenuation is aligned with the center frequency $\omega_{0}$ of the optical signal, and at this frequency, the residual attenuation is $2 \alpha_{R}$. In the analysis presented here, we assume that the wavelength dependence of the background attenuation is weak compared with the strong wavelength dependence of the attenuation in the hole. The hole in the attenuation translates, via Kramers-Kronig relationships, into a region centered on the peak of the hole, where the $n$ versus $\omega$ characteristic is approximately linear and has a large slope. One limitation of delay lines based on EIT and similar phenomena is that they are essentially bandpass devices. Thus, the nonconstant attenuation characteristic in Fig. 8(b) limits the bandwidth of the device.

\section{A. Ideal Slow-Light Delay Lines}

We now investigate the properties of the ideal device with refractive index in waveguide 2 as shown in Fig. 8(a). The objective here is to determine the ultimate limitations on slow-light propagation in an ideal device. In Sections III-B and III-C, we investigate the properties of practical slow-light devices using EIT/PO and microresonator-based devices.

The slope of the ideal effective refractive-index characteristic in Fig. 8(a) is

$$
\frac{d n_{2}}{d \omega}=\frac{2\left(n_{\mathrm{avg}}-n_{\min }\right)}{\omega_{\max }-\omega_{\min }} .
$$

To determine the ultimate limits on performance, we maximize this slope by matching $\left(\omega_{\max }-\omega_{\min }\right)$ to the signal bandwidth $\Delta \omega$, i.e., by putting $\left(\omega_{\max }-\omega_{\min }\right)=\Delta \omega$. We now derive an expression for the slow-down factor $S^{\text {ideal }}$. To do this, we put $d n_{1} / d \omega=0$ in (7) and substitute (8). If the fractional bandwidth $\Delta \omega / \omega_{0}$ of the signal is much smaller than unity, then $\omega \cong \omega_{0}$ and $n_{2}(\omega) \ll 2 \omega\left(n_{\text {avg }}-n_{\min }\right) / \Delta \omega$. Thus, (7) becomes

$$
S^{\text {ideal }}=\frac{2 c\left(n_{\mathrm{avg}}-n_{\min }\right)}{n_{1} \lambda_{0} \Delta v}
$$

where $\lambda_{0}$ is the free-space wavelength at the band center and $\Delta v=\Delta \omega / 2 \pi$ is the bandwidth of the optical signal in $\mathrm{Hz}$. The group velocity in waveguide 2 is obtained by putting $v_{g 1}=c / n_{1}$

$$
v_{g 2}=\frac{c}{S}=\frac{\lambda_{0} \Delta v}{2\left(n_{\mathrm{avg}}-n_{\mathrm{min}}\right)}=\frac{\lambda_{0} B_{\mathrm{packet}}}{\left(n_{\mathrm{avg}}-n_{\mathrm{min}}\right)} .
$$

Equation (10) shows that there is a tradeoff between the bandwidth of the data and the group velocity $v_{g 2}$. Increasing the data bandwidth $B_{\text {packet }}$ will always come at the price of increased group velocity.

We now calculate the storage time of the ideal Class A buffer. Storage time is determined by the maximum range of controllable delay. It was assumed that the group velocity in waveguide 2 can be changed from a maximum of $v_{g 1}$ (in which case, waveguide 2 becomes identical to waveguide 1) to a 
minimum of $v_{g 2}$. Under these conditions, the range of delay or storage time $T_{S}^{\text {ideal }}$ of an ideal device of length $L$ is

$$
T_{S}^{\text {ideal }}=L\left(\frac{1}{v_{g 2}}-\frac{1}{v_{g 1}}\right) .
$$

For $v_{g 2} \ll v_{g 1}$, the storage time is

$$
T_{S}^{\text {ideal }}=\frac{L}{v_{g 2}}=\frac{2 L\left(n_{\mathrm{avg}}-n_{\mathrm{min}}\right)}{\lambda_{0} \Delta v} .
$$

From (12), the delay-bandwidth product (i.e., the capacity) of the ideal delay line is

$$
C^{\text {ideal }}=T_{S}^{\text {ideal }} B_{\text {packet }}=\frac{L\left(n_{\text {avg }}-n_{\text {min }}\right)}{\lambda_{0}}
$$

and the capacity $C_{N}^{\text {ideal }}$ of the buffer per neper of loss is obtained by putting $L=1 / \alpha_{R}$

$$
C_{N}^{\text {ideal }}=\frac{n_{\text {avg }}-n_{\text {min }}}{\alpha_{R} \lambda_{0}}=\frac{1}{\alpha_{R} L_{\text {bit }}^{\text {ideal }}}
$$

where $L_{\text {bit }}^{\text {ideal }}$ is the size (i.e., the length) of each stored bit

$$
L_{\text {bit }}^{\text {ideal }}=\frac{L}{C^{\text {ideal }}}=\frac{\lambda_{0}}{\left(n_{\text {avg }}-n_{\min }\right)} .
$$

The maximum storage density $\mathrm{SD}^{\text {ideal }}$ of bits of data per unit length of the delay line is

$$
\mathrm{SD}^{\text {ideal }}=\frac{1}{L_{\text {bit }}^{\text {ideal }}}=\frac{\left(n_{\mathrm{avg}}-n_{\mathrm{min}}\right)}{\lambda_{0}} .
$$

Note that in the ideal device, since $L_{\mathrm{bit}}^{\text {ideal }}$ and $\mathrm{SD}^{\text {ideal }}$ are both independent of the bit rate, the capacity of the buffer is independent of bit rate. We show in Section III-B that this is not the case for EIT/PO devices. Equations (15) and (16) show that the storage density of an ideal slow-light device is related to the wavelength of the light. If $n_{\text {avg }} \gg n_{\min }$, then $\mathrm{SD}_{\text {max }} \approx n_{\mathrm{avg}} / \lambda_{0}$. In other words, the minimum possible size of a stored bit is approximately one wavelength in the waveguide. For example, if $n_{\text {avg }}=3.6, n_{\min }=1.5$, and $\lambda_{0}=$ $1.55 \mu \mathrm{m}$, then from (15) the minimum size of a stored bit is $L_{\text {bit }}^{\text {ideal }}=740 \mathrm{~nm}$. It is worth noting that this is in the order of the size of a state-of-the-art CMOS device, which suggests that slow-light buffers may be able to achieve storage densities that are comparable with storage densities in CMOS. However, CMOS technology continues to scale to smaller sizes while the bit size given by (15) is a fundamental limitation.

\section{B. EIT/PO Slow-Light Delay Lines}

This section derives an expression for the slow-down factor in active bandpass devices of the kind illustrated in Fig. 8(b) that use EIT, PO, and similar phenomena in atomic media. With $n=\operatorname{Re} \sqrt{\varepsilon}$, where $\varepsilon$ is the dielectric constant, (7) becomes

$$
S(\omega) \cong \frac{n_{2}}{n_{1}}+\frac{\omega}{2 n_{1} n_{2}} \frac{d \operatorname{Re} \varepsilon(\omega)}{d \omega} .
$$

The real part of $\varepsilon$ can be expressed in terms of the imaginary part of $\varepsilon$ via the Kramers-Kronig relationship

$$
\operatorname{Re} \varepsilon(\omega)=1+\frac{1}{\pi} \mathbf{P V} \int_{-\infty}^{\infty} \frac{\operatorname{Im} \varepsilon\left(\omega^{\prime}\right)}{\omega^{\prime}-\omega} \mathrm{d} \omega^{\prime}
$$

where PV represents the principal value. Substituting (17) into (18), we obtain

$$
S(\omega)^{\mathrm{EIT}} \cong \frac{n_{2}}{n_{1}}+\frac{1}{2 \pi n_{1} n_{2}} \mathbf{P V} \int_{-\infty}^{\infty} \frac{\operatorname{Im} \varepsilon\left(\omega^{\prime}\right)}{\left(\omega^{\prime}-\omega\right)^{2}} \mathrm{~d} \omega^{\prime} .
$$

Thus, the slow-down factor at the line center is given by

$$
S\left(\omega_{0}\right)^{\mathrm{EIT}}=\frac{n_{2}}{n_{1}}+\frac{1}{2 \pi n_{1} n_{2}} \mathbf{P V} \int_{-\infty}^{\infty} \frac{\operatorname{Im} \varepsilon(\omega)}{\left(\omega-\omega_{0}\right)^{2}} \mathrm{~d} \omega .
$$

The next step is to relate $\operatorname{Im} \varepsilon(\omega)$ in (20) to the attenuation. We define the optical frequency-dependent attenuation $\Delta \alpha(\omega)$ as the depth of the spectral hole in relation to the background attenuation. The maximum value of $\Delta \alpha$ is $\Delta \alpha\left(\omega_{0}\right)$ at the center frequency $\omega_{0}$, as shown in Fig. 8(b). As pointed out earlier, Fig. 8(b) also shows the residual attenuation $\alpha_{R}$ at the center frequency. We model the spectral hole by a simple Lorentzian as

$$
\Delta \alpha(\omega) \cong \frac{\Delta \alpha\left(\omega_{0}\right)}{1+\left(\frac{2 \delta}{\omega_{\frac{1}{2}}}\right)^{2}}
$$

where $\delta=\left(\omega-\omega_{0}\right)$ and $\omega_{1 / 2}$ is the full-width at halfmaximum (FWHM) linewidth of the spectral hole, as shown in Fig. 8(b). Note that Fig. 8(b) shows (with broken lines) that at the extremities of the attenuation characteristic, the actual characteristic will deviate from the assumed Lorentzian function. However, the $\operatorname{Im} \varepsilon(\omega)$ characteristic calculated from the Kramers-Kronig relationship is dominated by the $\Delta \alpha\left(\omega_{0}\right)$ characteristic in the hole, and deviations from the Lorentzian function well outside the passband have little effect on the result.

Substituting (21) into (20) with $\Delta \alpha(\omega)=-\omega n \operatorname{Im} \varepsilon(\omega) / c$ and $\omega_{1 / 2}=\Delta \omega$, we obtain an expression for the slow-down factor in terms of the linewidth and the attenuation at the center frequency of the spectral hole

$$
S\left(\omega_{0}\right)^{\mathrm{EIT}}=\frac{n_{2}}{n_{1}}+\frac{c \Delta \alpha\left(\omega_{0}\right)}{n_{1} \omega_{\frac{1}{2}}} .
$$

As with ideal Class A devices, the storage time for a device of length $L$ is determined by the change of group velocity. If the group velocity in waveguide 2 can be changed from a maximum of $v_{g 1}$ to a minimum of $v_{g 2}$, and if $v_{g 2} \ll v_{g 1}$, the delay time $T_{S}^{\mathrm{EIT}}$ of an EIT/PO-based device of length $L$ is $T_{S}^{\mathrm{EIT}}=L / v_{g 2}$. Thus, the delay is

$$
T_{S}^{\mathrm{EIT}}=L\left(\frac{n_{2}}{c}+\frac{\Delta \alpha\left(\omega_{0}\right)}{\omega_{\frac{1}{2}}}\right) .
$$


For devices with a large slow-down factor, the second term in (23) dominates over the first, and the delay-linewidth product $\mathrm{DL}^{\mathrm{EIT}}$ is [21]

$$
\mathrm{DL}^{\mathrm{EIT}}=T_{S}^{\mathrm{EIT}} \omega_{\frac{1}{2}}=L \Delta \alpha\left(\omega_{0}\right)
$$

Equation (24) shows that the delay-linewidth product is related to the length of the device and the depth of the spectral hole. Experimental verification of this simple relationship is presented in [14] for EIT in semiconductor quantum-well material. The delay-linewidth product per neper of loss $\mathrm{DL}_{N}^{\mathrm{EIT}}$ is obtained by putting $L=1 / \alpha_{R}$ in (24)

$$
\mathrm{DL}_{N}^{\mathrm{EIT}}=\frac{\Delta \alpha\left(\omega_{0}\right)}{\alpha_{R}} .
$$

We now consider signal transmission through the EIT/PO medium. For low-distortion signal transmission through an EIT/PO medium, the optical signal bandwidth $\Delta \omega$ should be less than or equal to the 3-dB bandwidth of the EIT/PO medium. To determine the $3-\mathrm{dB}$ bandwidth $\omega_{-3} \mathrm{~dB}$ of the medium, we write the optical power transfer function $\mathrm{TF}^{\mathrm{EIT}}$ of the EIT/PO medium in the form

$$
\operatorname{TF}^{\mathrm{EIT}}(\delta)=e^{-\left[\Delta \alpha\left(\omega_{0}\right)-\Delta \alpha(\delta)+2 \alpha_{R}\right] L} .
$$

The normalized transfer function $\operatorname{TF}^{\mathrm{EIT}}(\delta) / \mathrm{TF}^{\mathrm{EIT}}(0)$ has a magnitude of 0.5 when $2 \delta= \pm \omega_{-3} \mathrm{~dB}$. Thus, from (21) and (26)

$$
\ln (2)\left[\left(\frac{\omega_{\frac{1}{2}}}{\omega_{-3 \mathrm{~dB}}}\right)^{2}+1\right]=L \Delta \alpha\left(\omega_{0}\right)
$$

For large hole depth $\Delta \alpha\left(\omega_{0}\right),\left(\omega_{1 / 2} / \omega_{-3} \mathrm{~dB}\right)^{2} \gg 1$, and (27) can be simplified to

$$
\frac{\omega_{-3 \mathrm{~dB}}}{\omega_{\frac{1}{2}}}=\sqrt{\frac{\ln (2)}{L \Delta \alpha\left(\omega_{0}\right)}} .
$$

Equation (28) shows that the $-3-\mathrm{dB}$ bandwidth decreases with increasing length $L$. Substituting (28) in (24) and (25), and putting $\omega_{-3 \mathrm{~dB}}=4 \pi B_{\text {packet }}$, we obtain the delay-bandwidth product $C^{\mathrm{EIT}}$ (i.e., the capacity)

$$
C^{\mathrm{EIT}}=T_{S}^{\mathrm{EIT}} B_{\text {packet }}=\frac{\sqrt{\ln (2)}}{4 \pi} \sqrt{L \Delta \alpha\left(\omega_{0}\right)}
$$

the delay-bandwidth product per neper of loss $C_{N}^{\mathrm{EIT}}$ (with $\left.L=1 / \alpha_{R}\right)$

$$
C_{N}^{\mathrm{EIT}}=\frac{\sqrt{\ln (2)}}{4 \pi} \sqrt{\frac{\Delta \alpha\left(\omega_{0}\right)}{\alpha_{R}}}
$$

and the capacity-bandwidth product

$$
C^{\mathrm{EIT}} B_{\text {packet }}=\frac{\ln (2)}{(4 \pi)^{2}} \omega_{\frac{1}{2}} \cdot
$$

The size of a stored bit is

$$
L_{\mathrm{bit}}^{\mathrm{EIT}}=\frac{L}{C^{\mathrm{EIT}}}=\frac{4 \pi}{\sqrt{\ln (2)}} \sqrt{\frac{L}{\Delta \alpha\left(\omega_{0}\right)}}
$$

and the storage density $\mathrm{SD}^{\mathrm{EIT}}$ for EIT/PO devices is

$$
\mathrm{SD}_{\max }^{\mathrm{EIT}}=\frac{1}{L_{\mathrm{bit}}^{\mathrm{EIT}}}=\frac{\sqrt{\ln (2)}}{4 \pi} \sqrt{\frac{\Delta \alpha\left(\omega_{0}\right)}{L}} .
$$

Note that unlike the ideal devices considered in Section III-A above, the capacity and the bit size are proportional to $L^{1 / 2}$, and the storage density is proportional to $L^{-1 / 2}$. This square root dependency is caused by the fact that the bandwidth is reduced as $L$ increases. This bandwidth reduction with increasing length is an effect that is well known in cascaded filters. Note also that for a given EIT/PO material with fixed linewidth $\omega_{1 / 2}$ and fixed hole depth $\Delta \alpha\left(\omega_{0}\right)$, the capacity $C_{N}^{\mathrm{EIT}}$ per unit neper of loss and the capacity-bandwidth product $C^{\mathrm{EIT}} B_{\text {packet }}$ are independent of the buffer length $L$. We have recently verified the latter result using a numerical simulation of pseudorandom data in an EIT waveguide [22]. The capacity per neper of loss in an EIT/PO delay line is proportional to $\sqrt{\Delta \alpha\left(\omega_{0}\right) / \alpha_{R}}$. The implications of all of these results are discussed in Section III-D. Finally, note that the analysis above assumes that the EIT/PO material is uniformly pumped along its length. Typical pump power levels for EIT/PO devices are around 1-10 $\mathrm{mW}$ in tightly confined waveguides. These pump levels are easily achieved in practice, but there are many practical challenges to be overcome in achieving constant pump levels in the presence of losses along the waveguide.

\section{PC Microcavity Delay Lines}

We now consider passive slow-light devices based on PC waveguides incorporating passive microresonators. Early work on passive slow-wave waveguide structures dates back to the development of microwave travelling wave amplifiers [23]. Passive slow-wave structures are based on waveguiding in the presence of periodically spaced resonators. In recent years, a number of research groups have investigated slow-wave propagation at optical frequencies using Bragg grating structures [24], various forms of discrete microresonators [25], and resonator structures in PCs [16], [17]. Much of this work has been focused on reducing the group velocity in order to increase the optical field intensity to enhance nonlinear effects for applications such as switching and wavelength conversion [26].

The analysis presented here covers both passive and active PC structures. In passive PC structures, the microcavities are embedded in a passive dielectric material. In active PC delay lines, the microcavities are embedded in an active slow-light medium such as a material exhibiting EIT or PO. In essence, active devices combine the properties of slow-light materials with those of passive microstructure filters. It has recently been shown that devices of this kind can produce very long lifetimes in microresonator cavities [27]. The question we now address is, Do active PC delay lines provide improved slowlight buffering capabilities? We show that active PC delay lines 


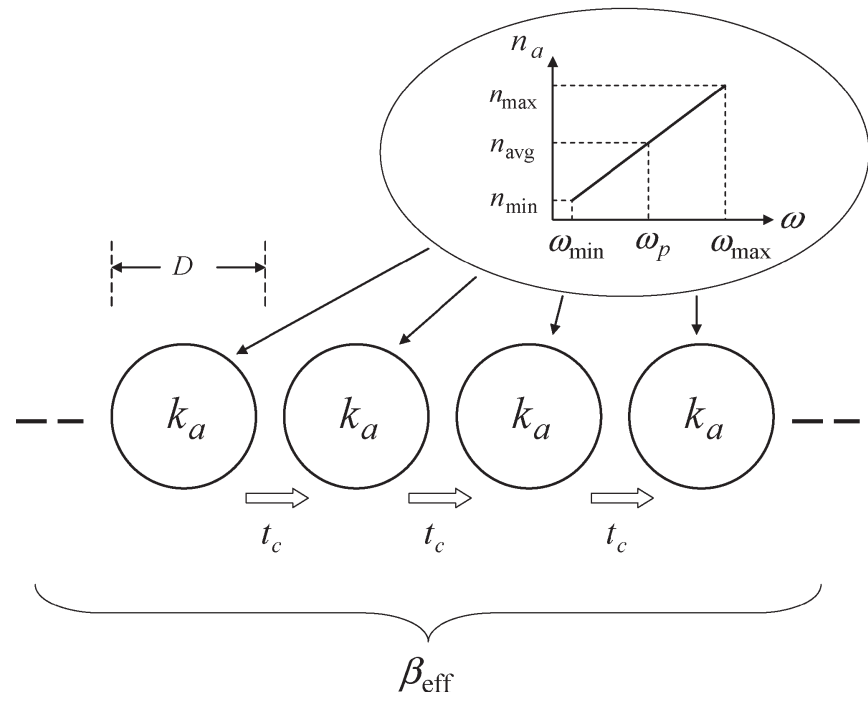

Fig. 9. Active microresonator delay line.

have the same fundamental limitations as passive PC delay lines, but active devices can approach the limitations more rapidly in practice.

Fig. 9 shows part of an active optical slow-light delay line consisting of a linear array of equally spaced optical microresonators. The resonators have dimension $D$, and the coupling coefficient between resonators is $t_{c}$. For simplicity, it is assumed that $t_{c}$ is independent of frequency and that the resonators can be approximated by Fabry-Pérot devices. We assume an infinitely long device and that there is no coupling between nonadjacent resonators. The material in each resonator is an active EIT or PO material. The active material is characterized in terms of its refractive index versus frequency profile. In the present analysis, the refractive index $n_{a}(\omega)$ of the active material in the resonators is assumed to have an ideal linear function of frequency, as shown in the inset in Fig. 9. The refractive index at the center of the region of linear refractive index is $n_{\text {avg }}$, and the center frequency $\omega_{p}$ of the region of linear refractive index is set to be identical to the center frequency of one of the cavity resonance passbands.

The propagation constant $k_{a}$ within each resonator is given by $k_{a}=\omega n_{a}(\omega) / c$, and the effective propagation constant $\beta_{\text {eff }}$ of a Bloch wave in the microresonator structure is $\beta_{\text {eff }}=$ $\omega n(\omega) / c$, where $n(\omega)$ is the (frequency dependent) effective refractive index of the microresonator structure in Fig. 9. The objective of the present analysis is to find $n(\omega)$ and to use this $n(\omega)$ to determine the properties of the delay line. A Blochwave analysis of the structure in Fig. 9 yields the dispersion characteristic [26]

$$
t_{c} \cos \left(\beta_{\mathrm{eff}} D\right)=\sin \left(k_{a} D\right)
$$

From (34), the effective propagation constant of the delay line is given by

$$
\beta_{\mathrm{eff}}(\omega)=\frac{1}{D}\left[2 q \pi \pm \cos ^{-1}\left(\frac{\sin \left(k_{a} D\right)}{t_{c}}\right)\right]
$$

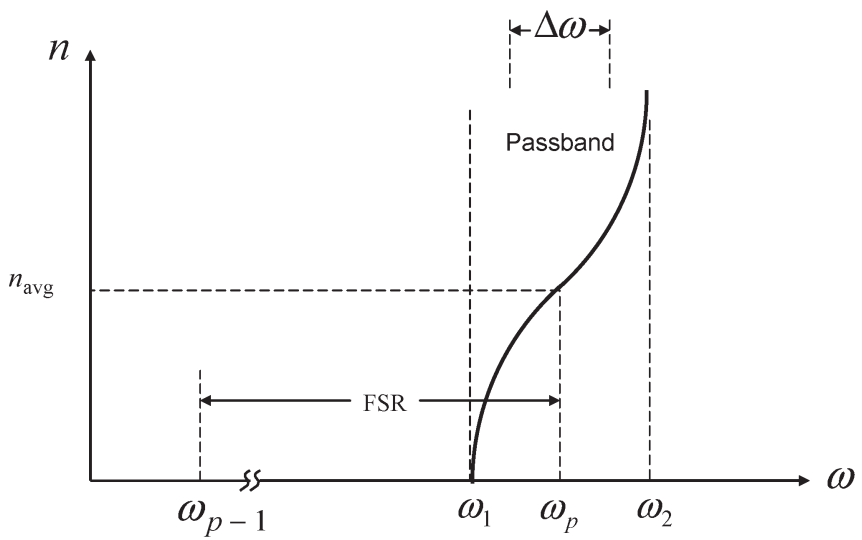

Fig. 10. Effective refractive versus frequency for coupled microresonators.

where $q$ is an integer. The passbands of the cavity resonances are centered at $k_{a} D=2 p \pi$ (i.e., at frequencies $\omega_{p}=$ $2 p \pi c / n_{\text {avg }} D$ ), where $p$ is an integer. The passbands are separated by the free spectral range [(FSR), in radians per second], where FSR $=2 \pi c / n_{\mathrm{avg}} D$. Thus, $\omega_{p}=p \mathrm{FSR}$.

To determine the slow-light properties of the delay line, it is necessary to obtain an expression for the bandwidth $\left(\omega_{2}-\omega_{1}\right)$ of each passband. The band edges $\omega_{1}$ and $\omega_{2}$ are frequencies where $\sin \left(k_{a} D\right) / t_{c}= \pm 1$. To find the bandwidth, we recognize that the relative bandwidth $\left(\omega_{2}-\omega_{1}\right) / \omega_{p}$ of the resonances is much less than unity. Thus, we put $\omega=\omega_{p}+\delta \omega$, where $\delta \omega$ is a small frequency deviation around the resonance frequency, and write $k_{a}$ in the form

$$
k_{a}=\frac{n_{\mathrm{avg}}}{c}\left[\omega_{p}+\delta \omega\left(1+\left(\omega_{p}+\delta \omega\right) \frac{d n_{a}}{d \omega}\right)\right]
$$

where $d n_{a} / d \omega=\left(n_{\max }-n_{\min }\right) /\left(\omega_{\max }-\omega_{\min }\right)$. From (36), it is easy to show that for small $\delta \omega / \omega_{p}$, the bandwidth of the passbands is given by

$$
\left(\omega_{2}-\omega_{1}\right) \cong \frac{2 c \sin ^{-1}\left(t_{c}\right)}{n_{\text {avg }} D\left(1+\omega_{p} \frac{d n_{a}}{d \omega}\right)} .
$$

Fig. 10 shows one passband in the effective refractive index $n(\omega)$ versus the frequency characteristic for $q=0$ and $p=1$. From (34), it follows that the effective refractive index $n\left(\omega_{p}\right)$ at the center of a passband is $n\left(\omega_{p}\right)=n_{\text {avg. }}$. The slope of effective propagation constant of the delay line as a function of frequency can be obtained by differentiating (34) to obtain

$$
\frac{d \beta_{\mathrm{eff}}(\omega)}{d \omega}=\frac{\cos (k D)}{\sqrt{t_{c}^{2}-\sin ^{2}(k D)}} \frac{d k_{a}}{d(\delta \omega)} .
$$

Note that the slope approaches infinity at the band edges, and the group velocity approaches zero at these frequencies. From (37) and (38), the slope at the band center $\left(\omega=\omega_{p}\right.$ and $k D=2 p \pi)$ is

$$
\left.\frac{d \beta_{\mathrm{eff}}(\omega)}{d \omega}\right|_{\omega=\omega_{p}}=\frac{1+\omega_{p} \frac{d n_{a}}{d \omega}}{t_{c}} .
$$


Thus, the group velocity $v_{g p}$ at the band center is given by

$$
v_{g p}=\frac{c}{n_{\mathrm{avg}}} \frac{1}{S_{\mathrm{PC}} S_{\mathrm{ACT}}}
$$

where $S_{\mathrm{PC}}=1 / t_{c}$ is the slow-down factor due to the resonators in the PC, and $S_{\mathrm{ACT}}=1+\omega_{p}\left(d n_{a} / d \omega\right)$ is the slow-down factor due to the active (EIT/PO) medium in the resonators. The key microresonator parameter affecting the group velocity is the coupling coefficient $t_{c}$. By controlling the coupling coefficient, the storage time can be changed [25]-[28]. Smaller coupling coefficients produce lower group velocities. One potential problem with PC-based slow-light devices is the difficulty of tuning the coupling coefficient $t_{c}$. Equation (40) shows that by combining passive and active slow-light techniques, the available controllable range of group velocity is greatly enhanced.

The storage time for an active PC device of length $L$ is

$$
T_{S}^{\mathrm{PC}}=\frac{L}{v_{g p}}=\frac{L n_{\mathrm{avg}}}{c} S_{\mathrm{PC}} S_{\mathrm{ACT}} .
$$

To calculate the delay-bandwidth product, it was assumed (as before) that the optical signal fully occupies the available bandwidth in the waveguide. For optimum device design, we assume that the EIT/PO medium has been selected such that its 3-dB bandwidth is at least as large as the bandwidth of the PC passbands. This ensures that the full bandwidth of the PC structure can be realized. From Fig. 10, it can be seen that signal components near the band edges may suffer some distortion through dispersion due to the nonideal (nonlinear) characteristic in Fig 10. In practical applications, it will be necessary to reduce the signal bandwidth to reduce this distortion to acceptable levels. However, we note that with appropriate filter design of the PC filter using a nonminimum phase structure [27] or using side resonators [29], a linear $n$ versus $\omega$ characteristic could be achieved over most of the passband. The design details of an optimized filter response are beyond the scope of the present paper. Instead, to obtain an upper estimate of the achievable delay-bandwidth product, we put $\left(\omega_{2}-\omega_{1}\right)=4 \pi B_{\text {packet }}$ and assume that the hold-off time $T_{\mathrm{HO}}$ is equal to the packet length $\tau_{p}$. Thus, the delay-bandwidth product (or capacity) is

$$
C^{\mathrm{PC}}=T_{S}^{\mathrm{PC}} B_{\text {packet }}=\frac{T_{S}^{\mathrm{PC}}\left(\omega_{2}-\omega_{1}\right)}{4 \pi} .
$$

From (37) and (41), this becomes

$$
C^{\mathrm{PC}}=\frac{L \sin ^{-1}\left(t_{c}\right)}{2 \pi t_{c} D} .
$$

For a large slow-down factor $S_{\mathrm{PC}}, t_{c} \ll 1$ and $\sin ^{-1}\left(t_{c}\right) \cong t_{c}$ [26]. Thus, the delay-bandwidth product becomes

$$
C^{\mathrm{PC}}=\frac{L}{2 \pi D}=\frac{N_{\text {res }}}{2 \pi}
$$

where $N_{\text {res }}=L / D$ is the number of resonators. The delay-bandwidth product per neper of loss is

$$
C_{N}^{\mathrm{PC}}=\frac{1}{2 \pi \alpha_{R} D}=\frac{1}{\alpha_{R} L_{\mathrm{bit}}^{\mathrm{PC}}}
$$

and the bit size $L_{\mathrm{bit}}^{\mathrm{PC}}=L / C^{\mathrm{PC}}$ is

$$
L_{\mathrm{bit}}^{\mathrm{PC}}=2 \pi D .
$$

Equation (46) shows that the minimum bit size is limited by the dimension $D$ of the microresonators. Thus, microresonator filters can achieve higher storage density than delay-line filters with larger resonators [24]. The minimum achievable $D$ is limited by the resonator resonance condition $\omega_{p}=p \pi c /\left(n_{\mathrm{avg}} D\right)$. The minimum value $D$ is achieved when $p=1$, i.e., when the filter is operated at the first resonance passband. With $p=1$, (44) and (46) become

$$
C^{\mathrm{PC}}=\frac{L n_{\mathrm{avg}}}{\pi \lambda_{0}}
$$

and

$$
L_{\mathrm{bit}}^{\mathrm{PC}}=\frac{\pi \lambda_{0}}{n_{\mathrm{avg}}} .
$$

The storage density is

$$
\mathrm{SD}_{\max }^{\mathrm{PC}}=\frac{n_{\mathrm{avg}}}{\pi \lambda_{0}} .
$$

Equation (48) shows that when $p=1$, the minimum size of a stored bit is approximately equal to the wavelength of the optical signal in the waveguide. Note that this lower singledimensional limit on the size of a stored bit is approximately the same as the limitation for an ideal device, with a linear effective index versus frequency characteristic, as given in (15), depending on the value of $n_{\min }$ in (15). For comparison, in three-dimensional space, light in PCs can be confined within a volume in the order of $(\lambda / 2 n)^{3}[30]$.

\section{Capabilities and Limitations of Class A Slow-Light Buffers}

Equations (10)-(16), (24)-(33), and (40)-(49) contain useful information about capabilities, limitations, and design tradeoffs for Class A low-light optical buffers. Equations (13), (29), and (44) give the delay-bandwidth product for an ideal slow-light device, an EIT/PO device with a Lorentzian-shaped attenuation, and a PC device, respectively; (14), (30), and (45) give the delay-bandwidth products per neper of loss for an ideal slow-light device, an EIT/PO, and PC devices; and (31) gives the capacity-bandwidth product for an EIT/PO device. These results are summarized, along with some other key results, in Table I.

Some key functional dependencies highlighted in Table I are as follows. The capacity of ideal devices and PC devices increases linearly with buffer length, but the capacity of EIT/PO buffers increases as the square root of length. This square-root dependency arises because the bandwidth is compressed as the 
TABLE I

Theoretical Limitations on Storage Time, Delay-Bandwidth Product, Minimum Bit Size, Maximum Storage Density, and

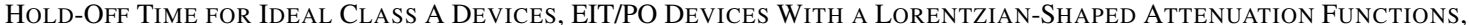
PC Devices as Defined in the TeXt, Class B Devices, And Combined A/B/A Devices as Shown in Fig. 16(a). $\mathrm{T}_{R}$ IS THE RECONFIGURATION TIME FOR GROUP-VELOCITY CHANGE

\begin{tabular}{|c|c|c|c|c|c|c|c|c|}
\hline \multicolumn{2}{|c|}{ Class } & \multirow{2}{*}{$\begin{array}{c}\begin{array}{c}\text { Delay } \\
(\text { Storage Time) }\end{array} \\
\frac{L\left(n_{\text {avg }}-n_{\min }\right)}{\lambda_{0} B_{\text {packet }}}\end{array}$} & \multirow{2}{*}{$\begin{array}{c}\begin{array}{c}\text { Delay-Bandwidth } \\
\text { Product }\end{array} \\
\frac{L\left(n_{\text {avg }}-n_{\min }\right)}{\lambda_{0}}\end{array}$} & \multirow{2}{*}{$\begin{array}{c}\begin{array}{c}\text { Delay-Bandwidth } \\
\text { Product/Neper Loss }\end{array} \\
\frac{n_{\mathrm{avg}}-n_{\min }}{\alpha_{R} \lambda_{0}}\end{array}$} & \multirow{2}{*}{$\begin{array}{c}\text { Delay- } \\
\text { Capacity } \\
\text { Product }\end{array}$} & \multirow{2}{*}{$\begin{array}{l}\text { Min Bit Size } \\
\frac{\lambda_{0}}{\left(n_{\text {avg }}-n_{\min }\right)}\end{array}$} & \multirow{2}{*}{$\begin{array}{l}\text { Storage Density } \\
\frac{\left(n_{\mathrm{avg}}-n_{\min }\right)}{\lambda_{0}}\end{array}$} & \multirow{2}{*}{$\begin{array}{l}\text { Hold-Off Time } \\
\qquad T_{S}+T_{R}\end{array}$} \\
\hline A & Ideal & & & & & & & \\
\hline A & $\mathrm{EIT} / \mathrm{PO}$ & $\frac{\sqrt{\ln (2)}}{4 \pi B_{\text {packet }}} \sqrt{L \Delta \alpha\left(\omega_{0}\right)}$ & $\frac{\sqrt{\ln (2)}}{4 \pi} \sqrt{L \Delta \alpha\left(\omega_{0}\right)}$ & $\frac{\sqrt{\ln (2)}}{4 \pi} \sqrt{\frac{\Delta \alpha\left(\omega_{0}\right)}{\alpha_{R}}}$ & $\frac{\ln (2)}{(4 \pi)^{2}} \omega_{1 / 2}$ & $\frac{4 \pi}{\sqrt{\ln (2)}} \sqrt{\frac{L}{\Delta \alpha\left(\omega_{0}\right)}}$ & $\frac{\sqrt{\ln (2)}}{4 \pi} \sqrt{\frac{\Delta \alpha\left(\omega_{0}\right)}{L}}$ & $T_{S}+T_{R}$ \\
\hline A & $\mathrm{PC}$ & $\frac{L n_{0}}{c t_{c}}=\frac{L n_{\mathrm{avg}}}{\pi \lambda_{0} B_{\mathrm{packet}}}$ & $\frac{L n_{\text {avg }}}{\pi \lambda_{0}}$ & $\frac{n_{\mathrm{avg}}}{\pi \alpha_{R} \lambda_{0}}$ & & $2 \pi D=\frac{\pi \lambda_{0}}{2 n_{\text {avg }}}$ & $\frac{1}{2 \pi D}=\frac{2 n_{\text {avg }}}{\pi \lambda_{0}}$ & $T_{S}+T_{R}$ \\
\hline \multicolumn{2}{|c|}{ B (Ideal) } & $S\left(\frac{L}{v_{g 1}}-T_{R}-\tau_{p}\right)$ & $S\left(\frac{L}{L_{\mathrm{bit}}^{\text {input }}}-C^{\text {Class B }}\right)$ & $S\left(\frac{1}{\alpha_{R} L_{\mathrm{bit}}^{\text {input }}}-C^{\text {Class B }}\right)$ & & $\frac{c \tau_{b}}{n_{1}}$ & $\frac{n_{1}}{c \tau_{b}}$ & $T_{S}+T_{R}+2 S \tau_{p}$ \\
\hline \multicolumn{2}{|c|}{$\begin{array}{c}\mathrm{A} / \mathrm{B} / \mathrm{A} \\
\text { (Ideal) } \\
\text { Fig. 16(a) }\end{array}$} & $S \tau_{p}\left(\frac{L\left(n_{\text {avg }}-n_{\min }\right)}{\lambda_{0}}-C^{\text {Class } \mathrm{B}}\right)$ & $S\left(\frac{L\left(n_{\text {avg }}-n_{\min }\right)}{\lambda_{0}} C^{\text {Class } \mathrm{B}}\right)$ & $S\left(\frac{n_{\mathrm{avg}}-n_{\min }}{\alpha_{R} \lambda_{0}}-C^{\text {Class } \mathrm{B}}\right)$ & & $\frac{\lambda_{0}}{\left(n_{\text {avg }}-n_{\min }\right)}$ & $\frac{\left(n_{\mathrm{avg}}-n_{\min }\right)}{\lambda_{0}}$ & $T_{S}+T_{R}+2 S \tau_{p}$ \\
\hline \multicolumn{2}{|c|}{$\begin{array}{l}\text { Fiber Plus } \\
\text { Crosspoint }\end{array}$} & $\frac{L n_{\text {fiber }}}{c}$ & $\frac{L n_{\text {fiber }}}{c \tau_{b}}$ & $\frac{n_{\text {fiber }}}{c \tau_{b} \alpha_{R}}$ & & $\frac{c \tau_{b}}{n_{\text {fiber }}}$ & $\frac{n_{\text {fiber }}}{c \tau_{b}}$ & $T_{S}+T_{R}$ \\
\hline
\end{tabular}

device length is increased. The physical length of a stored bit in an ideal device is independent of the buffer length. This results from the assumption that each device has been optimized so that its bandwidth is matched to the signal bandwidth. The capacity per neper of loss in all Class A devices is independent of the buffer length, and the capacity-bandwidth product in EIT devices is independent of the buffer length. Finally, for a given buffer length and fixed material properties, the delay-bandwidth product in all Class A devices is fixed. Thus, for a fixed device length, the delay can be increased by decreasing the bandwidth (or bit rate) and vice versa.

The delay-linewidth product of an EIT/PO slow-light device as given in (24) can never exceed the delay-bandwidth product of the ideal device in (12) and (13). By equating $T_{S}^{\text {ideal }} \Delta v$ in (12) to the delay-linewidth product in (24), the maximum usable value of $\Delta \alpha\left(\omega_{0}\right)$ for slow light is $2\left(n_{\text {avg }}-\right.$ $\left.n_{\min }\right) / \lambda_{0}$. For example, if $n_{\text {avg }}=3.6$ and $n_{\min }=2.0$, the maximum achievable value of $\alpha\left(\omega_{0}\right)$ at a wavelength of $\lambda_{0}=1.55 \mu \mathrm{m}$ is $2.1 \times 10^{6} \mathrm{~m}^{-1}$. In recent demonstrations of slow light, values of $\Delta \alpha\left(\omega_{0}\right)$ of this order have been achieved using EIT in atomic vapor cells [12] and quantum-well material [16].

As pointed out earlier, the minimum size of a stored data bit $L_{\text {bit }}^{\text {ideal }}$ in an ideal slow-light device is related to the wavelength of the light. For example, with $n_{\text {avg }}=3.6, n_{\min }=2.0$, and $\lambda_{0}=1.55 \mu \mathrm{m}$, the size of a stored bit is $L_{\text {bit }}^{\text {ideal }}=740 \mathrm{~nm}$. By comparison, in [12], the size of each equivalent stored bit at an equivalent bit rate of $150 \mathrm{~kb} / \mathrm{s}$ was $L_{\mathrm{bit}} \sim 110 \mu \mathrm{m}$ for a device length of $L=330 \mu \mathrm{m}$, and in [16] the size of each equivalent stored bit at an equivalent bit rate of $1 \mathrm{~Gb} / \mathrm{s}$ was $L_{\mathrm{bit}} \sim 10 \mu \mathrm{m}$ for a device length of $L=0.43 \mu \mathrm{m}$. From (29), the values of $\Delta \alpha\left(\omega_{0}\right)$ in [12] and [16] are approximately $1 \times 10^{6}$ and $2.3 \times 10^{6}$, respectively. The available bandwidth in [12] is relatively small $(\sim 150 \mathrm{kHz})$ while the group velocity is also very small $(17 \mathrm{~m} / \mathrm{s})$. On the other hand, in [16], the available bandwidth $(\sim 2 \mathrm{GHz})$ is about four orders of magnitude larger than in [12], and the group velocity $(9600 \mathrm{~m} / \mathrm{s})$ is about three orders of magnitude larger. This highlights the fundamental tradeoff between group velocity and bandwidth in slow-light devices.

The minimum size of a stored bit $L_{\mathrm{bit}}^{\mathrm{PC}}$ in a PC slow-light device is given in (46). This simple expression shows that the minimum size of a stored bit is approximately six times the spacing of the microresonators. This fundamental limit can be thought of as an aliasing limit imposed by the sampling-trainlike eigenmodes of the waveguide [31]. As pointed out above, the lower limit on $L_{\mathrm{bit}}^{\mathrm{PC}}$ in (48) is closely related to the limitation for an ideal device, as given in (15). An important conclusion here is that the maximum delay-bandwidth product and the minimum size of a stored bit in a PC slow-light delay line is the same whether or not the delay line is active. In other words, using slow-light material (e.g., EIT or PO) in the cavities does not change the ultimate limitations on storage capacity. However, as shown in (41), the slow-down factor is enhanced by using slow-light material in the resonators. Therefore, active PC devices will make it easier in practice to approach the ultimate limitations provided the bandwidth of the EIT/PO material is sufficiently broad.

As pointed out earlier, the delay-bandwidth product (or capacity) per neper $(8.7 \mathrm{~dB})$ of loss provides a measure of the loss-induced limitations on buffer capacity. Equations (14), (30), and (45) show that this quantity is independent of device length and is maximized for low residual attenuation $\alpha_{R}$. For a residual loss of $0.5 \mathrm{~dB} / \mathrm{cm}$ (typical of an integrated planar waveguide), the capacity of an ideal device with the parameters given above, the capacity per neper of loss calculated using (14) is $C_{N}^{\text {ideal }}=235 \mathrm{~kb}$. In other words, an ideal buffer with a residual loss of $0.5 \mathrm{~dB} / \mathrm{cm}$ and a total loss of $8.7 \mathrm{~dB}$ (i.e., a length of $L=17.4 \mathrm{~cm}$ ) could store $235 \mathrm{~kb}$ of data. As shown earlier, the physical size of each stored bit would be $740 \mathrm{~nm}$. For an EIT device with the same residual loss $(0.5 \mathrm{~dB} / \mathrm{cm})$ and $\Delta \alpha\left(\omega_{0}\right)=2 \times 10^{6}$ (typical of an EIT device), the capacity from (30) is $C_{N}^{\mathrm{EIT}}=39 \mathrm{~b}$ and the size of each stored bit is $L_{\mathrm{bit}}^{\mathrm{EIT}}=L / C_{N}^{\mathrm{EIT}}=4.5 \mathrm{~mm}$. The capacity per 
neper of loss in an EIT/PO delay line is proportional to $\sqrt{\Delta \alpha\left(\omega_{0}\right) / \alpha_{R}}$. Thus, the device capacity is maximized by maximizing the ratio $\Delta \alpha\left(\omega_{0}\right) / \alpha_{R}$. If the residual loss in decibels could be reduced by two orders of magnitude to around $0.005 \mathrm{~dB} / \mathrm{cm}$ (perhaps by incorporating optical gain in the device), the capacity for the ideal device and the EIT device would increase to $23.5 \mathrm{Mb}$ and $391 \mathrm{~b}$, respectively.

It is useful to compare the storage density of slow-light devices with the density of stored bits in a fiber delay line incorporating an optical switch or cross point [see Fig. 2(b)]. For a fiber delay line, the size of each stored bit is

$$
L_{\mathrm{bit}}^{\mathrm{fiber}}=\frac{c \tau_{b}}{n}
$$

and the storage density is $1 / L_{\mathrm{bit}}^{\mathrm{fiber}}$. If the data rate is $40 \mathrm{~Gb} / \mathrm{s}$, and the refractive index $n$ is 1.5 , then the size of a stored bit is $L_{\mathrm{bit}}^{\mathrm{fiber}}=0.5 \mathrm{~cm}$. One striking advantage of optical fiber compared with the other buffer media considered here is its exceptionally low loss. At a data rate of $40 \mathrm{~Gb} / \mathrm{s}$, a fiber with a loss of $0.2 \mathrm{~dB} / \mathrm{km}$ (1 Np of loss for a length of $43.5 \mathrm{~km}$ ) has a single-wavelength storage capacity of $8.7 \mathrm{Mb}$ or 37 times the capacity of an ideal slow-wave device with a loss of $0.5 \mathrm{~dB} / \mathrm{cm}$ and $2.2 \times 10^{5}$ times the capacity of the EIT device in the previous paragraph, also with a loss of $0.5 \mathrm{~dB} / \mathrm{cm}$. The fiber delay line would be $2.5 \times 10^{5}$ times longer than the EIT device. At $40 \mathrm{~Gb} / \mathrm{s}$, the storage density in the fiber $(200 \mathrm{~b} / \mathrm{m})$ is approximately the same as the storage density in the EIT device $(222 \mathrm{~b} / \mathrm{m})$ and $0.015 \%$ of the storage density in the ideal slowlight device $\left(1.35 \times 10^{6} \mathrm{~b} / \mathrm{m}\right)$. At $160 \mathrm{~Gb} / \mathrm{s}$, the fiber would have approximately four times the storage density as the EIT device. An important conclusion here is that the size advantage of slow-light delay lines over fiber-based delay lines is reduced as the bit rate increases.

As pointed out in Section II, in all buffers the rate at which packets can be loaded into each delay line is limited by the hold-off time. The hold-off time for a Class A delay line is the time required to clear the buffer of data and to change the group velocity to a new value. Thus, the hold-off time $T_{\mathrm{HO}}$ is

$$
T_{\mathrm{HO}}=T_{C}+T_{R} \approx T_{C}
$$

where $T_{C}$ is the time to clear the buffer of the packet or group of packets and $T_{R}$ is the reconfiguration time required to change the group velocity of the delay line. In many cases, $T_{C}$ is equal to the storage time $T_{C} . T_{R}$ is on the order of the coherence lifetime for EIT devices ( $1 \sim 100 \mathrm{ps}$ for semiconductors), the carrier lifetime for PO devices ( $\sim 1 \mathrm{~ns}$ for semiconductors), and $1 \sim 100 \mathrm{~ns}$ for PC waveguide devices (see Section III-C), depending on the tuning mechanism for the refractive index. Note that the hold-off time can never be less than the packet length. Otherwise, data will be corrupted. Finally, it is worth noting that the capacity and/or functionality of all the devices mentioned previously can be increased by combining a number of basic buffers either in cascade or in series provided, of course, that optical losses can be contained.

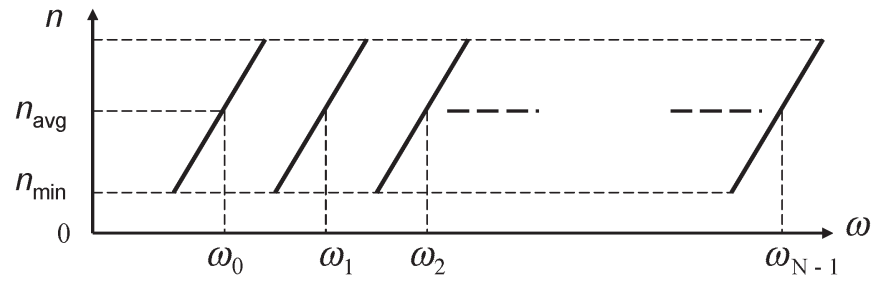

Fig. 11. WDM slow-light characteristics.

\section{E. Wavelength-Multiplexed Class A Delay Lines}

It is possible, in principle, to increase the capacity of a delayline buffer by WDM [1]. This approach to increased capacity comes at the cost of increased complexity associated with wavelength multiplexing and demultiplexing components. In addition, the need for separate control of each wavelength channel introduces limitations. Nevertheless, it is useful to consider the possible improvement in buffer performance with WDM. Fig. 11 shows the refractive index against the optical frequency for an ideal WDM buffer. In Fig. 11, there are $N$ wavelength channels, centered at optical frequencies $\omega_{i}, i=0, N-1$. With $N$ optical channels, the capacity and storage density of a buffer are increased by a factor of $N$. One possible approach to achieving the characteristic in Fig. 11 would be to make use of multiple passbands in a PC device. The principal difficulty with this approach is that relatively large resonators would be needed in order to achieve reasonable wavelength band spacings, and this would limit the buffer capacity, according to (44). A second approach would be to leverage nonuniformity in an array of quantum dots and to use different pump laser wavelengths to excite each different group of quantum dots.

\section{Buffers With Time-Dependent Group VELOCITY (Class B)}

In this section, we consider delay lines in which the groupvelocity profile $v_{g}(x, t)$ in (1) can change with time while data are stored in the delay line. Tunable group-velocity reduction of this kind was first proposed in [28] and was used experimentally in [13] to bring light to a virtual standstill. The technique was further investigated using simulations in [29] for a slow-wave PC structure with side resonators. To our knowledge, there has been no reported demonstration of slow-light propagation in PCs with tuned group velocity. In the present paper, we label these tunable group-velocity slow-light devices "Class B" delay lines. Fig. 12 shows how a cascade of $M$ Class B delay lines could be used to construct a FIFO buffer. In this example, the group velocity in each of the $M$ delay lines can be adjusted continuously between an upper limit of $v_{g 1}$ and a lower limit of $v_{g 2}$ using the control signals $\zeta_{1}(t)-\zeta_{M}(t)$. The FIFO buffer operates as follows. An incoming packet enters the first delay line (DL1) at velocity $v_{g 1}$. Once the entire packet has entered DL1, the group velocity in DL1 is changed adiabatically from $v_{g 1}$ to $v_{g 2}$. The first of the two insets in Fig. 12 shows this deceleration with time. In the inset, the deceleration is uniform, but we will show later that nonuniform deceleration is permissible. The group velocity is $v_{g 2}$ in all delay lines between the most recently accepted 


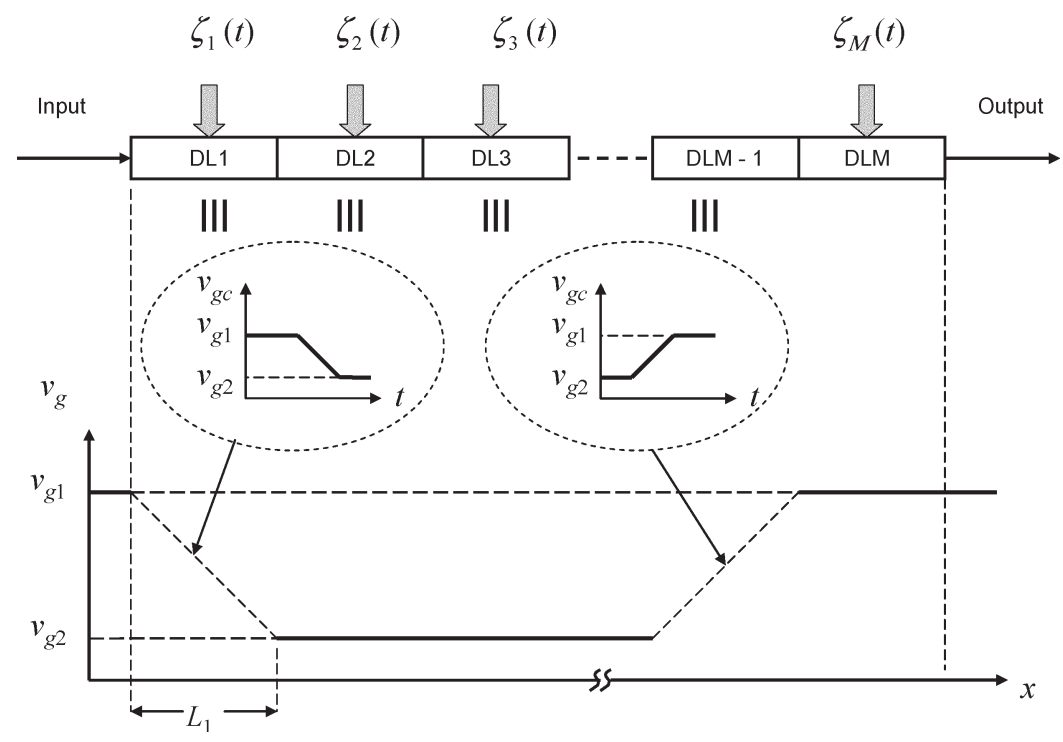

Fig. 12. FIFO buffer using Class B delay lines.

packet and the packet closest to the output. When it is required to read the packet closest to the output, the group velocity in the delay line where the packet is located is ramped back to $v_{g 1}$ as shown in the second inset.

\section{A. Tuning Characteristics}

We now look in detail at how Class B delay lines affect the propagation properties of stored packets. Fig. 13 shows the effective-index of an ideal Class B device. Fig. 13 is a "snapshot" of the effective refractive index taken at one point in time while the group velocity is changing. The time dependence of the group velocity is caused by a rotation of the linear effective-index profile about the point "x" in Fig. 13. In Fig. 13, we have selected the point of rotation to coincide with the average refractive index $n_{\text {avg }}$. However, other points could be selected, such as point "y," which corresponds to the geometric mean $\sqrt{n_{\min } n_{\max }}$ of the extreme values of the refractive index. Note that regardless of the point of rotation, as the angle $\theta$ changes, the average refractive index $n_{\text {avg }}$, the minimum refractive index $n_{\min }$, and the maximum refractive index $n_{\max }$ all remain unchanged. A clockwise rotation about " $x$ " produces an increase of the group velocity, and an anti-clockwise rotation produces a decrease in group velocity.

As pointed out earlier, there has been speculation in the literature [28], [29] that Class B delay lines could be constructed using microresonator-based PC waveguide filters with the group velocity tuned by changing the coupling coefficient $t_{c}$ between coupled resonators. As shown in (39), tuning $t_{c}$ causes the slope of $\beta$ and the effective refractive index to change, and thus $t_{c}$ controls the group velocity. The viability of this technique has yet to be proven in practice, one difficulty being possible technology limits on the tuning range over which $t_{c}$ can be controlled. Another possibility for constructing Class B waveguides would be to use EIT/PO with the slope of the refractive-index profile being tuned by adjustment of the attenuation constant $\alpha\left(\omega_{0}\right)$. As shown in Section III-D above, a

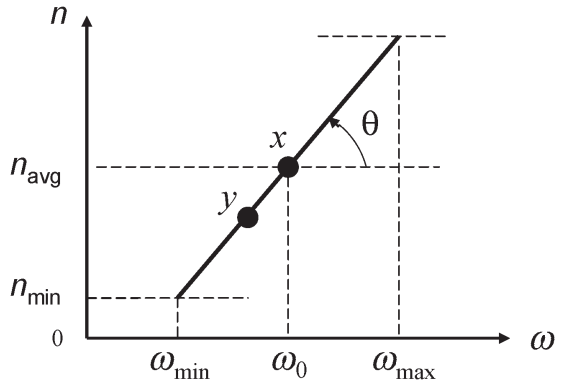

Fig. 13. Characteristics of Class B slow-light delay line.

combination of tuned microresonators and EIT/PO may help to overcome limitations on tuning range.

We now analyze the behavior of a device with timedependent refractive index. We assume that the time scale $\tau_{n}$ of the changes of refractive index is very much larger than the time scale of the optical carrier $\tau_{c}=1 / v$, i.e., assume that $\tau_{n} \gg \tau_{c}$. (This assumption is equivalent to the adiabatic assumption in [29].) Under this assumption, the variables $\omega$ and $t$ can be separated. From Fig. 13, the refractive index at frequency $\omega$ and time $t$ can be expressed in terms of the width $\left(\omega_{\max }-\omega_{\min }\right)$ of the region of linear refractive slope

$$
n(\omega, t)=n_{\mathrm{avg}}+\frac{2\left(n_{\mathrm{avg}}-n_{\min }\right)\left(\omega-\omega_{0}\right)}{\omega_{\max }-\omega_{\min }} .
$$

Thus

$$
\frac{d n(\omega, t)}{d t}=\frac{n_{\mathrm{avg}}-n(\omega, t)}{\omega_{\max }-\omega_{\min }} \frac{d \Delta \omega(t)}{d t}
$$

We now consider wave propagation through the Class B device from time $t$ to time $t+d t$. Using (5) and substituting (53), it is easy to show that

$$
E(t+d t, x)=E_{o} e^{j\left(\left(\omega+\omega_{S}\right)(t+d t)-k x\right)}
$$




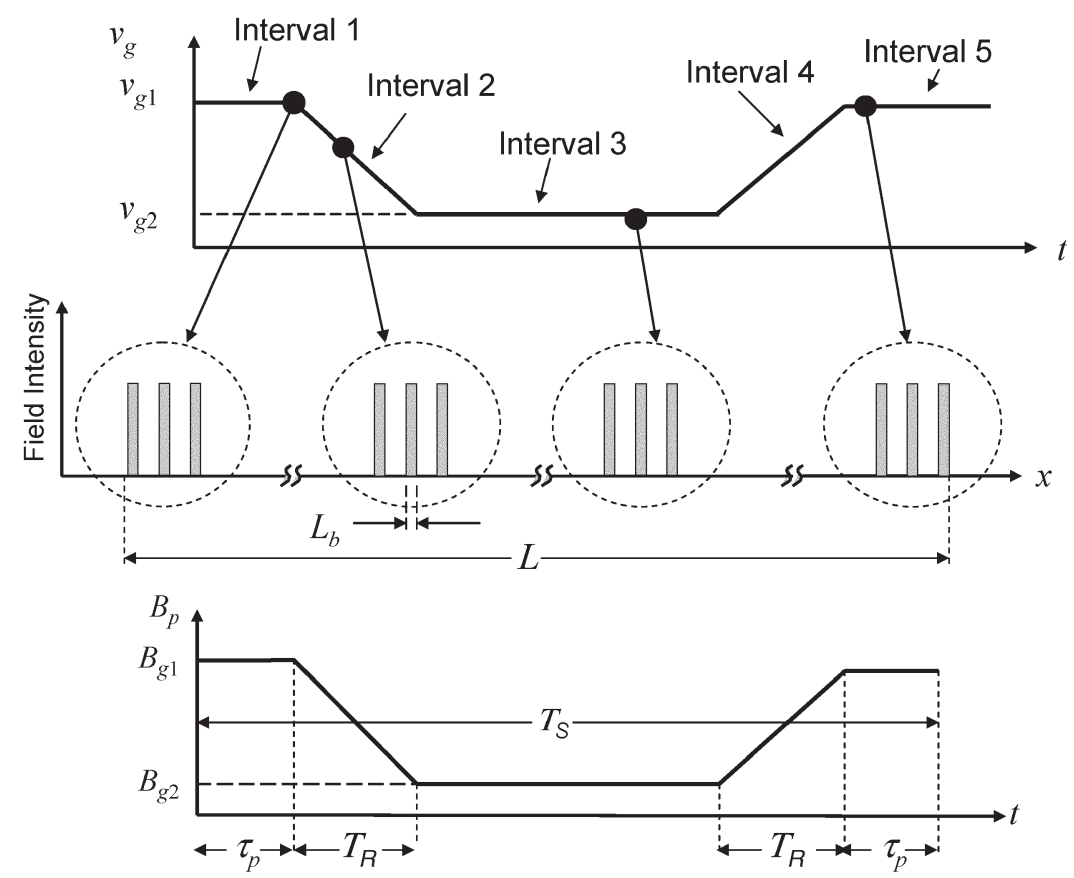

Fig. 14. Pulses in Class B slow-light variable delay line with time-dependent uniform group-velocity distribution.

where

$$
\omega_{s}=\left(\omega-\omega_{0}\right) \frac{d\left(\omega_{\max }-\omega_{\min }\right)}{\omega_{\max }-\omega_{\min }}
$$

is the frequency shift experienced by the component of the signal spectrum at frequency $\omega$.

Equations (54) and (55) show that for changes of refractive index that are very much slower than the time scale of the optical carrier, a rotation of the refractive-index profile shown in Fig. 13 causes a frequency shift $\omega_{s}$ of the optical signal. This frequency shift is a function of the frequency $\omega$ and is proportional to $\left(\omega-\omega_{0}\right)$, the spacing from the center frequency $\omega_{0}$. In other words, a small increase in the refractive-index slope and a corresponding fractional reduction $d\left(\omega_{\max }-\omega_{\min }\right) /$ $\left(\omega_{\max }-\omega_{\min }\right)$ in the width $\left(\omega_{\max }-\omega_{\min }\right)$ of the region of linear refractive index causes the bandwidth of the signal to be compressed by the same fractional amount. This means that the bandwidth of the signal tracks changes in the width $\left(\omega_{\max }-\right.$ $\left.\omega_{\min }\right)$ of the region of linear refractive index. Note that the signal is compressed at a rate that tracks the refractive-index profile regardless of the particular waveform of $\left(\omega_{\max }-\omega_{\min }\right)$ as a function of time. In the examples presented here, the slope of the index is a linear function of time, but other waveforms produce the same result.

Fig. 14 illustrates the characteristics of a Class B buffer of the type shown in Fig. 15. In Fig. 14, we focus on the time dependence of group velocity and bandwidth. In Fig. 14, a packet of three pulses enters the delay line and initially propagates at a group velocity of $v_{g 1}$. This continues for time $\tau_{p}$, during which the entire packet is loaded into the delay line. The interval during which the packet enters the delay line is designated Interval 1. After the packet is loaded, the group velocity decreases with time. This is designated (a)

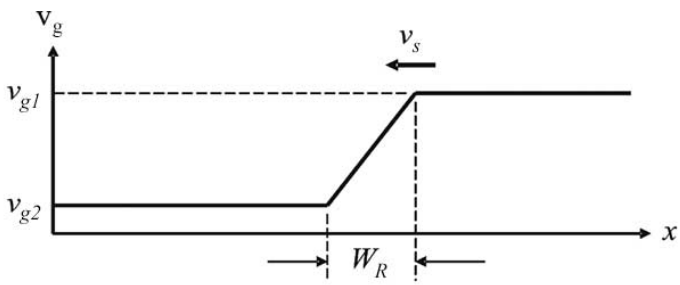

(b)

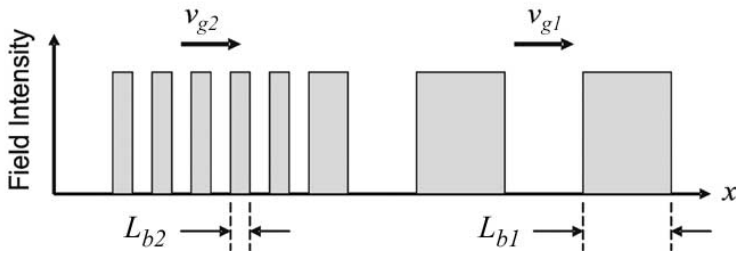

Fig. 15. Pulses in Class B slow-light variable delay line with moving velocity transition.

Interval 2, which is the "reconfiguration" period, during which the group velocity of the packet is reduced. The reconfiguration time is $T_{R}$. In Fig. 14, the group velocity decreases as a linear function of time in Interval 2. However, as pointed out previously, other (nonlinear) functions are possible provided the adiabatic condition is satisfied. Because the group-delay profile across the length of the delay line remains uniform, all bits in the packet see the same group velocity at any instant in time. This applies during Interval 2 and at all other times. After Interval 2, the group velocity becomes constant at a value of $v_{g 2}$ (Interval 3). In Interval 4 , the group velocity increases again and becomes constant again in Interval 5 .

The time dependence of $v_{g}$ is shown at the top of Fig. 14, the time dependence of bandwidth is shown at the bottom of Fig. 14, and four snapshots of the 3-b packet are shown at various points on the time evolution of $v_{g}$. The first snapshot is at the end Interval 1, when the packet has been fully loaded 
into the delay line, the second is in Interval 2, and so on. As pointed out above, all bits in the packet (and all parts of each bit) see the same group velocity at each instant in time. Thus, the data bits and the packets are not compressed in space as is the case in Class A delay lines. The length of a bit $L_{b}$ as shown in Fig. 12 is unchanged across the entire length of the delay line. Note that unlike Class A delay lines, the optical field in the pulses is unchanged along the entire length of the delay line.

Because the group velocity is smaller during Interval 3 than in Interval 1, and because of the invariance of the spatial dimensions of the bits and packets, it is easy to see that the bandwidth of the data is compressed during Interval 2 and is expanded during Interval 4 . The data bandwidth $B_{\text {packet }}^{\text {Interval }}$ in Interval 3 is given by $B_{\text {packet }}^{\text {Interval } 3}=B_{\text {packet }}^{\text {Interval } 1} / S$, where $S=v_{g 1} / v_{g 2}$ is the slow-down factor and $B_{\text {packet }}^{\text {Interval } 1}=$ $B_{\text {packet. }}$. From this, it can be seen that both the bandwidth and the bit rate of the data are decreased in inverse proportion to the slow-down factor. Similarly, the data bandwidth in Interval 5 is given by $B_{\text {packet }}^{\text {Interval } 5}=S B_{\text {packet }}^{\text {Interval } 3}$ and the output bandwidth $B_{\text {packet }}^{\text {output }}=B_{\text {packet }}^{\text {Interval } 5}$ is equal to the input bandwidth $B_{\text {packet }}$. It is important to point out that the average bandwidth (i.e., the effective throughput) of the buffer is limited by the bandwidth in Interval 3. While the input and output bandwidths are larger than this, the input and output data can occur only in short bursts due to the relatively long hold-off time.

As with Class A buffers, the storage time of Class B buffers is determined by the maximum range of controllable delay. For the buffer illustrated in Fig. 14, it is easy to show that for $S \gg 1$ the storage time $T_{S}^{\text {Class B }}$ is

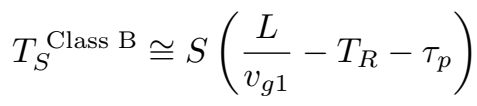

where the reconfiguration time $T_{R}$ is the minimum ramp time for the group velocity, as shown in Fig. 14. Note that for a given device length and slow-down factor, the storage time is maximized when the ramp time $T_{R}$ and the packet length $\tau_{p}$ are minimized. The delay-bandwidth product is

$$
T_{S}^{\text {Class B }} B_{\text {packet }}=\frac{S}{\tau_{b}}\left(\frac{L}{v_{g 1}}-T_{R}-\tau_{p}\right) .
$$

Note that the delay and the delay-bandwidth product are greater than zero only if $L / v_{g 1}>T_{R}+\tau_{p}$. The device will function only if this condition is satisfied. This means that the device length must be at least equal to the length of the incoming packet on the input line plus the propagation distance of the packet during reconfiguration.

If $T_{R} \ll \tau_{p}$, the delay-bandwidth product can be written as

$$
T_{S}^{\text {Class B }} B_{\text {packet }}=S\left(\frac{L}{L_{\text {bit }}^{\text {input }}}-b_{\text {packet }}\right)
$$

where $L_{\mathrm{bit}}^{\text {input }}=v_{g 1} \tau_{p}$ is the size of a bit in the input packet as it enters the delay line and $b_{\text {packet }}=C^{\text {Class B }}$ is the number of bits in the input packet or the number of bits stored (i.e., the capacity of the buffer). The capacity of the buffer can be obtained from (58) as

$$
C^{\text {Class B }}=\frac{L}{L_{\text {bit }}^{\text {input }}}-\frac{T_{S}^{\text {Class B }} B_{\text {packet }}}{S} .
$$

For large slow-down factor $S$, the capacity becomes

$$
C^{\text {Class B }} \approx \frac{L}{L_{\text {bit }}^{\text {input }}} .
$$

The capacity per neper of loss is

$$
C_{N}^{\text {Class B }} \approx \frac{1}{\alpha_{R} L_{\text {bit }}^{\text {input }}} .
$$

From (58), it can be seen that the device will not function unless $L / L_{\text {packet }}^{\text {input }}>b_{\text {packet. }}$ In other words, Class B devices require large $L$ and/or short packets.

The size of a stored bit $L_{\mathrm{bit}}^{\text {Class B }}$ on the delay line (i.e., during Interval 3) is equal to the size $L_{\mathrm{bit}}^{\text {input }}$ of the bits as they enter the buffer. Therefore, the storage density of bits in a Class B buffer is

$$
\mathrm{SD}^{\text {Class B }}=\frac{C^{\text {Class B }}}{L} \approx \frac{1}{L_{\mathrm{bit}}^{\text {input }}} .
$$

Thus, the minimum bit size and the average storage are, at best, equal to the size and the storage density of bits on the input waveguide. This means that the minimum bit size in Class B buffers is much larger than for Class A buffers. Finally, the hold-off time $T_{\mathrm{HO}}^{\text {Class B }}$ in a Class B device is

$$
T_{\mathrm{HO}}^{\text {Class B }} \cong T_{S}^{\text {Class B }}+T_{R}+S \tau_{p} .
$$

Equations (56)-(63) highlight some important characteristics of Class B buffers. Unlike Class A buffers, there is no fundamental upper limit on the delay-bandwidth product for a Class B buffer. However, also unlike Class A buffers, the delay-bandwidth product can approach zero if the device is short or if the input packet length is large. Like Class A buffers, the delay time in Class B buffers $T_{S}^{\text {Class B }}$ can be made large by trading off the bandwidth $B_{\text {packet }}$. A major disadvantage of Class B buffers is that they suffer from a very severe limitation on the minimum size of stored bits. This is because in Class B devices, the stored bits are not compressed.

\section{B. Capabilities and Limitations of Class B Slow-Light Buffers}

To obtain a feel for the capabilities and limitations of Class B devices, we consider the simulation results presented in [29] for a Class B delay line. In [29], the bit rate is $250 \mathrm{~Gb} / \mathrm{s}$, and the bit period is $\tau_{b}=4 \mathrm{ps}$. Since there is no size compression of the bits in a Class B device, the bit size in the low-velocity region is $0.8 \mathrm{~mm}$ (assuming an input waveguide with a refractive index of 1.5). This bit size is the same as in a passive waveguide with the same average effective refractive index. This corresponds to a storage density of only 12 bits per centimeter of device length. For a short device, the average storage density would be even less than this because a significant length of short devices 
is required at the input of the delay line for loading the input data during Interval 1 [see Fig. 14 and (57)].

One key advantage of Class B delay lines is that the delay-bandwidth product can in principle be made very large. In fact, since the delay-bandwidth product is proportional to the slow-down factor $S$, as shown in (58), there is no fundamental limitation on the delay-bandwidth product. However, in Class B devices, a large delay-bandwidth product does not imply a large capacity, as shown in (59). For example, the device simulated in [29] has a slow-down factor of $10^{4}$ and the effective bandwidth during the low-velocity Interval 3 is $25 \mathrm{MHz}$. If the simulated device in [29] has a residual loss of $0.5 \mathrm{~dB} / \mathrm{cm}$, the capacity per neper of device loss is $281 \mathrm{~b}$. If the bit rate of the stored data was $40 \mathrm{~Gb} / \mathrm{s}$, the capacity of the device per neper would be only $35 \mathrm{~b}$. This means that Class B buffers, by themselves, are not suited to large-capacity applications such as OPS. We explore this issue in more detail in Section VI.

In essence, the reason for the poor storage density of Class B devices is that the entire packet to be stored needs to enter the buffer in Interval 1 at the group velocity of the input waveguide. When the input packet is decelerated to group velocity $v_{g 2}$, the physical size of the bits is not decreased. This means that the storage density in Interval 3 is also equal to the storage density the input waveguide.

\section{Alternative Realization of Class B Delay Line}

Fig. 15 shows another possible realization of a Class B delay line. In this figure, we concentrate on the output portion of the delay line, in which a slow-light packet is accelerated back to full velocity. Fig. 15(a) shows a snapshot of a pulse train moving to the right at group velocity $v_{g 2}$ and accelerating to group velocity $v_{g 1}$. The group-velocity profile in the delay line has a ramp increase in group velocity from $v_{g 2}$ to $v_{g 1}$. The width $W_{R}$ of the ramp is constant and the (constant) slope of the ramp is $S_{R}=\left(v_{g 1}-v_{g 2}\right) / W_{R}$. The distinguishing feature of this particular delay line is that the location of the ramp increase in group velocity is itself moving along the delay line. In the case we consider here, the ramp is moving to the left with a velocity of $v_{s}$.

If the velocity $v_{s}$ of the position of the step is comparable to the group velocity $v_{g 2}$, then the time taken for a pulse to cross the step is less for a stationary step. Thus, the bit period of the outgoing pulses is decreased, and the bit rate is increased. From Fig. 15, it easy to show that the ratio of the length of the outgoing pulses $L_{b 1}$ to the length $L_{b 2}$ of the incoming pulses is

$$
\frac{L_{b 1}}{L_{b 2}}=\frac{v_{s}+v_{g 1}}{v_{s}+v_{g 2}}
$$

and the ratio of the bit rate $B_{\text {out }}$ of the outgoing pulses to the bit rate $B_{\text {in }}$ of the bit rate of the ingoing pulses is

$$
\frac{B_{\text {out }}}{B_{\text {in }}}=\frac{L_{b 2}}{L_{b 1}} \frac{v_{g 1}}{v_{g 2}} .
$$

Note that when the transition in group velocity is stationary (i.e., when $v_{s}=0$ ), the output bit rate is identical to the input (a)

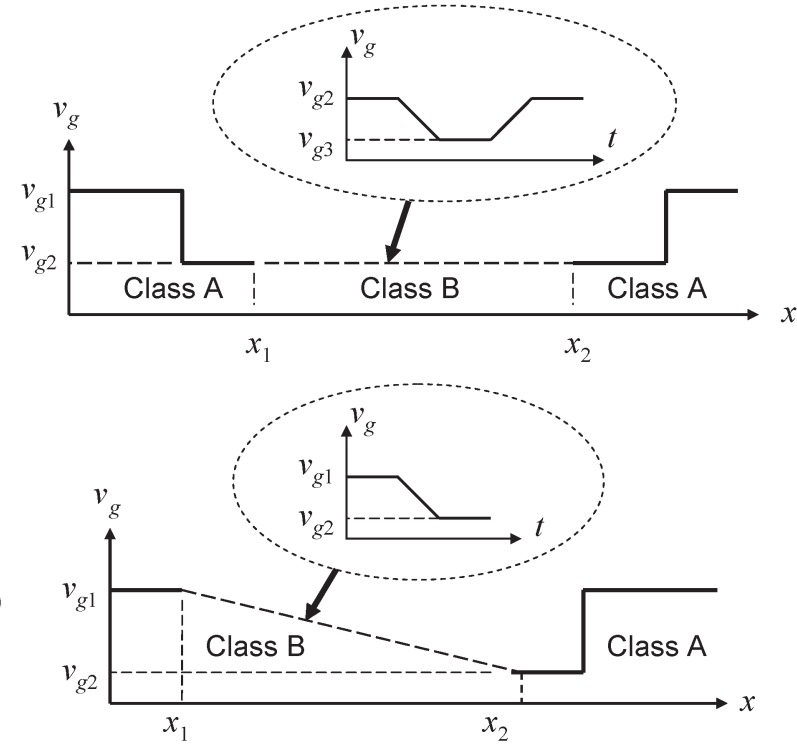

Fig. 16. Mixed delay lines. (a) Increased slow-down factor. (b) Packet decompressor.

bit rate. In this case, the device is a Class A device. In the other extreme, when the velocity $v_{s}$ of the transition is large, then $B_{\text {out }}=S B_{\text {in }}$. Note that this bandwidth increase is identical to the bandwidth increase in a device with a uniform groupvelocity distribution that ramps in time (Section IV-A). The example considered in Fig. 15 is for a ramp increase in group velocity moving to the left. If the ramp is changed from an increase to a decrease in group velocity (from $v_{g 1}$ to $v_{g 2}$ ), then it is easy to show that $B_{\text {out }}=B_{\text {in }} / S$.

\section{Mixed Delay Lines (Class AB)}

A wide variety of buffering and signal processing functions can be envisioned if Class A and Class B devices are cascaded. Two examples are shown in Fig. 16. Fig. 16(a) shows the group-velocity distribution for a buffer comprising a Class B device sandwiched between two Class A devices. We call this an $\mathrm{A} / \mathrm{B} / \mathrm{A}$ buffer. The group velocity on the incoming waveguide is $v_{g 1}$. This is slowed to $v_{g 2}$ by the first Class A device. The slowed data at $v_{g 2}$ is then injected into a Class B device that is located between positions $x_{1}$ and $x_{2}$. Because the group velocity in the Class B device changes with time, it is shown with a broken line in between $x_{1}$ and $x_{2}$ [Fig. 16(a)]. The inset in Fig. 16(a) shows the time evolution of group velocity in the Class B device. The Class B device slows the data to a group velocity of $v_{g 3}$, stores the data for some time period, and then accelerates the data back to $v_{g 2}$. Finally, the second Class A device restores the data to its original group velocity $v_{g 1}$.

One advantage of the cascaded A/B/A buffer in Fig. 16(a) is that it can potentially provide a combined slow-down factor that is larger than either of the two component devices. More significantly, the arrangement in Fig. 16(a) relieves the limitations on storage density in Class B devices. By slowing the incoming packets in a Class A device, the incoming packets and bits are compressed to the same physical size as in an ideal Class A device. This greatly reduces the length of the Class B device that needs to be set aside for loading the incoming packet 
(Interval 1). It also increases the storage density in the Class B device. In addition to achieving the storage density of a Class A device, the cascaded $\mathrm{A} / \mathrm{B} / \mathrm{A}$ device can in principle achieve the same (unbounded) delay-bandwidth product as an ideal Class B device. The storage time $T_{S}^{\mathrm{IdealABA}}$ in the $\mathrm{A} / \mathrm{B} / \mathrm{A}$ device is obtained by putting $v_{g 1}=\lambda_{0} B_{\text {packet }} /\left(n_{\text {avg }}-n_{\text {min }}\right)$ in (56) as

$$
T_{S}^{\text {IdealABA }}=\frac{S}{B_{\text {packet }}}\left(\frac{L\left(n_{\text {avg }}-n_{\text {min }}\right)}{\lambda_{0}}-C^{\text {Class B }}\right)
$$

where we have assumed that $T_{R}$ is small. Expressions for the delay-bandwidth product and other properties of $\mathrm{A} / \mathrm{B} / \mathrm{A}$ devices are summarized in Table I.

As an example, we consider the Class B device simulated in [29] with an ideal Class A device at the input that slows the group velocity and compresses the input data bits before they enter the Class B device. Another ideal Class A device accelerates the bits back to full speed at the output. The ideal Class A devices could be quite short because all the buffering occurs in the Class B device. From the previous discussion in Section III, the size of the input bits could be reduced in size to around $L_{\text {bit }}^{\text {input }}=740 \mathrm{~nm}$ by the ideal Class A device at the input (depending on the value of the average refractive index). Thus, from (61), the capacity per neper of the overall device (neglecting the short Class A input and output sections) would be the same as the capacity per neper of an ideal device-i.e., around $235 \mathrm{~kb}$. As before, the total capacity could be increased by combining a number of these Class A/ Class B devices either in cascade or in parallel. However, the capacity of the buffer would ultimately be limited by optical losses.

Another application of slow-light devices is in data or packet compression and expansion. Fig. 16(b) shows a packet decompressor using a Class B device cascaded with a Class A device. This decompressor takes advantage of bandwidth (and bit rate) slow-down provided by Class B devices. The Class B device in Fig. 16(b) is located between positions $x_{1}$ and $x_{2}$. It accepts a packet at group velocity $v_{g 1}$ and slows it to $v_{g 2}$. As in Fig. 14, the (unspecified) $x$ dependence of group velocity is shown with a broken line in Fig. 16(b). The time-dependent ramp in group velocity is shown in the inset. The slowed packet at position $x_{2}$ has a reduced bandwidth and bit rate. This reduced bitrate packet is then input to the Class $\mathrm{A}$ device at point $x_{2}$. The Class A device returns the packet to group velocity $v_{g 1}$ but does not change the bandwidth or bit rate. Therefore, the packet emerging from the output has a reduced bit rate but travels at the same group velocity as the input (high bit rate) packet. By turning the device around and ramping the group velocity up rather than down in the Class A device, the cascaded device becomes a packet compressor, in which the bit rate is increased rather than decreased. These packet compression and decompression devices may prove useful in a range of applications in high-speed communications and signal processing, including multiplexers, demultiplexers, and true FIFO buffers in which the read-out data rate can be different from the input data rate.

\section{Some Applications of Slow-Light Delay Lines}

This section considers the requirements on Class A slowlight delay lines for a number of applications.

\section{A. Optical Packet Switching (OPS)}

We first consider Class A delay lines in OPS. Applications of delay lines in OPS include buffering of data for contention avoidance, packet synchronization, and delaying of incoming buffers to allow the header to be fully read before a routing decision is made [1]-[5]. Returning to the example presented in the Section I, a 1000-port router with fiber-delay-line buffers having a storage capacity of around $4 \mathrm{~Gb}$ at each $40-\mathrm{Gb} / \mathrm{s}$ port would require a total length of fiber of approximately $40 \mathrm{Gm}$ or $4 \mathrm{Mm}$ per port. For reasons enunciated earlier, this amount of fiber would be quite impractical.

If the fibers were replaced by ideal slow-light devices with ideal (minimum) sizes of a stored bit of around $1 \mu \mathrm{m}$, the total length of the buffer waveguides could, in principle, be reduced by a factor of $5 \times 10^{3}$ to a total length of $8 \mathrm{Mm}$, or $8 \mathrm{~km}$ per port. If efficient buffering and networking schemes can be devised [9] to reduce the required buffering time per port by around two orders of magnitude, these numbers would fall to $80 \mathrm{~km}$ and $80 \mathrm{~m}$, respectively. At present, it is difficult to see how lengths of this magnitude could become practical using integrated circuit optical chips due to the relatively high optical loss in integrated waveguides compared with optical fiber. If we imagine that optical networking strategies may one day be developed that would enable a reduction in the required buffering capacity per port by five orders of magnitude below the $4 \mathrm{~Gb}$ used in present-day electronic routers, the lengths would reduce to $80 \mathrm{~m}$ and $8 \mathrm{~cm}$, respectively. At this level, slowlight buffering may become feasible in OPS. However, it is not clear that this level of buffering will ever be sufficient in highly loaded networks.

While slow-light devices do not appear practical for contention resolution in OPS, slow-light delay lines may provide feasible solutions to the problem of optical packet synchronization. The maximum delay required in a packet synchronizer is on the order of one packet length or about $1 \mu \mathrm{s}$ at $40 \mathrm{~Gb} / \mathrm{s}$. In an ideal device with bit sizes of around $1 \mu \mathrm{m}$, the length of a packet synchronization buffer would be around $0.3 \mathrm{~mm}$. In EIT material with $\Delta \alpha\left(\omega_{0}\right)=2 \times 10^{6}$, (29) shows that a device length of $11.3 \mathrm{~m}$ would be required. Without a mechanism for overcoming losses, this length would be impractical. However, by combining EIT and PC media, it may be possible to reduce the length to a reasonable value.

\section{B. True Time Delay for Microwave Systems}

True time delay lines find application in microwave systems such as antenna beam-forming networks [8] that require continuously variable delays. Miniaturization is a key requirement especially when many tens or hundreds of delay lines are required in a single system such as a phased-array antenna. Variable microwave delay lines can be constructed from optical delay lines with microwave signals subcarrier multiplexed onto optical carriers. The microwave delay of an ideal microwave 
TABLE II

Examples of EfFective Bandwidth, Delay-Bandwidth Product, Bit Size, Capacity, and $\Delta \alpha\left(\omega_{0}\right)$

\begin{tabular}{|c|c|c|c|c|c|c|}
\hline \multicolumn{2}{|c|}{ Class } & Bandwidth & $\begin{array}{l}\text { Delay-Bandwidth } \\
\text { Product }\end{array}$ & Bit Size & $\begin{array}{c}\text { Capacity/neper } \\
@ 0.5 \mathrm{~dB} / \mathrm{cm}\end{array}$ & $\Delta \alpha\left(\omega_{0}\right)$ \\
\hline A & Ideal & & $1.4 \times 10^{4 ~ \#, *}(\mathrm{IIID})$ & $740 \mathrm{~nm}{ }^{\#}$ (IIID) & $235 \mathrm{~kb}$ (all bit rates) & $\sim 2 \times 10^{6 \#}(\mathrm{IIID})$ \\
\hline A & EIT/PO & $\begin{array}{c}300 \mathrm{kHz}[12] \\
2 \mathrm{GHz}[14]\end{array}$ & $\begin{array}{l}2^{\wedge}[12] \\
<1^{\wedge}[14]\end{array}$ & $\begin{array}{l}110 \mu \mathrm{m} \wedge \text { [12] } \\
10 \mu \mathrm{m} \wedge[14]\end{array}$ & $39 \mathrm{~b}^{\wedge}[14]$ & $\begin{array}{c}\sim 10^{6}[12] \\
\sim 2 \times 10^{6}[16]\end{array}$ \\
\hline A & PC & $10 \mathrm{GHz}$ [24] & $168^{\wedge}[27]$ & $20 \mu \mathrm{m}^{\wedge}[27]$ & & \\
\hline \multicolumn{2}{|r|}{ B } & $250 \mathrm{GHz}$ [29] & $10^{4^{\wedge}, *}[29]$ & $0.8 \mathrm{~mm} \wedge[29]$ & $281 b^{\wedge}[29]$ & \\
\hline \multicolumn{2}{|c|}{$\begin{array}{c}\text { Ideal A/B/A } \\
\text { (Fig. 16(a)) }\end{array}$} & $250 \mathrm{GHz}$ [29] & & $740 \mathrm{~nm}^{\#}(\mathrm{IIID})$ & $235 \mathrm{~kb}$ (all bit rates) & \\
\hline \multicolumn{2}{|c|}{$\begin{array}{c}\text { Fiber/ } \\
\text { crosspoint }\end{array}$} & & $2^{\#, * * * *}$ (IIID) & $0.5 \mathrm{~cm}^{\#, * *}$ & & \\
\hline \multicolumn{2}{|c|}{ CMOS/RAM } & & Very Large & $\sim 100 \mathrm{~nm}^{2}$ & & \\
\hline
\end{tabular}

"Fundamental limit

^Example only - not a fundamental limit

* For a 1-cm long device

** $40 \mathrm{~Gb} / \mathrm{s}$ data

*** Not including Demux/Mux

delay line is equal to the slow-light optical delay and can be calculated from (13) simply by replacing $B_{\text {packet }}$ in (13) by the microwave carrier frequency (i.e., the spacing between the optical carrier and each of the subcarrier modulation sidebands). Thus, from (13) and using the same ideal material parameters as in the previous example, an ideal slow-light delay line that provides a 1-ns tunable range of delay for a microwave signal at $10 \mathrm{GHz}$ would need to be only a mere $7.4 \mu \mathrm{m}$ in length. In EIT material with $\Delta \alpha\left(\omega_{0}\right)=2 \times 10^{6}$, a device length of $11.4 \mathrm{~cm}$ would be required. This opens up the possibility of an entire beam-forming network on a single photonic chip.

\section{Optical Signal Processing}

Optical delay lines are key components for analog optical signal processing [4] and digital optical signal processing systems such as optical header processors [3]. As always, miniaturization is a key requirement. As shown earlier, an ideal slow-light buffer and an EIT device have loss-limited capacities of $235 \mathrm{~kb}$ and $39 \mathrm{~b}$, respectively, for a residual attenuation of $0.5 \mathrm{~dB} / \mathrm{cm}$. EIT devices with these characteristics would be suitable in signal processing applications where only short packets or bit streams need to be stored. One application that might be appropriate is in the area of header processing in OPS. The 39-b capacity limit on EIT devices could be increased to higher levels by improving the residual loss and combining PC waveguides with the EIT material.

\section{CONCLUSION}

We have developed a theoretical framework for quantifying the capabilities and limitations of controllable-delay slow-light optical delay lines. The analysis has been carried out for delay lines in which the delay properties can be changed only when no data are stored (Class A delay lines) and delay lines in which the delay properties can be changed while data are in the device (Class B delay lines). We show that there are important differences between these two classes of device and that in ideal devices optimum storage density and storage time can be achieved by combining Class A and Class B devices.

Some of the key theoretical limitations derived in this paper are summarized in Table I, which gives the storage time, the delay-bandwidth product (i.e., the capacity), the loss-limited capacity (capacity per neper of loss), the capacity-bandwidth product, the minimum size of a stored bit, the maximum storage density, and the hold-off time. These fundamental limitations are presented for ideal Class A delay lines, EIT/PO Class A delay lines, and active PC microresonator Class A delay lines, as well as ideal Class B delay lines and ideal Class B delay lines embedded in ideal Class A delay lines. Also included, for comparison, are fiber delay lines where controllability is provided via a crosspoint (see Fig. 2).

There is an upper limit on the delay-bandwidth product for all Class A delay lines. For ideal delay lines, the maximum achievable storage density is approximately $1 \mathrm{~b}$ per unit wavelength. In other words, the minimum size of a stored bit is one wavelength in the device waveguide. This ideal result applies PC-based microresonator buffers when the resonators become very small but has not been realized in any practical devices. EIT/PO devices can, in principle, approach the ideal storage density of $1 \mathrm{~b}$ per unit wavelength, but this can only be achieved for very short devices, where the capacity is small. The losslimited capacity of a delay line is a useful measure of the practical limit on capacity. The loss-limited capacity of an ideal slow-light delay line with an exceptionally low residual attenuation of $0.05 \mathrm{~dB} / \mathrm{cm}$ is only $2.35 \mathrm{Mb}$. For practical residual attenuations, which are likely to be larger than this, the losslimited capacity is even smaller. We conclude that slow-light buffers appear unlikely to be suitable for contention resolution in OPS, in which much larger capacities are needed. Slow-light buffers are more likely to find applications in systems requiring small but compact all-optical buffering.

Class B delay lines offer the potential of very slow group velocity and large storage times. However, this comes at the cost of storage density that is limited by the size of the bits on the input waveguide. In addition, Class B devices have a 
significant hold-off time or dead time during which additional data cannot be loaded into the device. This large hold-off time results in reduced effective bandwidth. We have shown that by combining Class A and Class B devices, one can, in principle, obtain the best of both worlds, including the large delay-bandwidth products that can be achieved with Class B devices and the high storage densities that can be obtained with Class A devices.

Table II summarizes some numerical values of the effective bandwidth, bit size, and $\alpha\left(\omega_{0}\right)$ for some examples from the literature and from this paper. The numbers in square brackets are reference numbers, and the numbers in curved brackets are section numbers from this paper. Note that the numbers in Table II are examples of results that have either been calculated or demonstrated experimentally. These numbers are summarized here for comparison purposes, but some of them do not necessarily represent the theoretical limitations. Finally, we note that the analysis presented here has ignored distortion due to dispersion and the question of optical pump requirements for active EIT/PO devices. The chief objective has been to determine fundamental limitations and to show some fundamental limitations of slow-light optical buffers. Further research on the design of low-distortion delay lines will assist in the design of practical buffers.

\section{REFERENCES}

[1] W. De Zhong and R. S. Tucker, "Wavelength routing-based photonic packet buffers and their applications in photonic packet switching systems," J. Lightw. Technol., vol. 16, no. 10, pp. 1737-1745, Oct. 1998.

[2] S. Yao, B. Mukherjee, and S. Dixit, "Advances in photonic packet switching: An overview," IEEE Commun. Mag., vol. 38, no. 2, pp. 84-94, Feb. 2000.

[3] H. J. S. Dorren et al., "Optical packet switching and buffering by using all-optical signal processing methods," J. Lightw. Technol., vol. 21, no. 1, pp. 2-12, Jan. 2003

[4] T. Sakamoto, A. Okada, M. Hirayama, Y. Sakai, O. Moriwaki, I. Ogawa, R. Sato, K. Noguchi, and M. Matsuoka, "Optical packet synchronizer using wavelength and space switching," IEEE Photon. Technol. Lett., vol. 14, no. 9, pp. 1360-1362, Sep. 2002.

[5] J. Spring and R. S. Tucker, "Photonic $2 \times 2$ packet switch with input buffers," Electron. Lett., vol. 29, no. 3, pp. 284-285, Feb. 4, 1993.

[6] D. B. Hunter and R. A. Minasian, "Photonic signal processing of microwave signals using and active-fiber Bragg-grating-pair structure," IEEE Trans. Microw. Theory Tech., vol. 45, no. 8, pp. 1463-1466, Aug. 1977.

[7] J. Capmany, J. Cascon, D. Pastor, and B. Ortega, "Reconfigurable fiberoptic delay line filters incorporating electrooptic and electroabsorption modulators," IEEE Photon. Technol. Lett., vol. 11, no. 9, pp. 1174-1176, Sep. 1999

[8] W. D. Jemison, T. Yost, and P. R. Herczfeld, "Acoustooptically controlled true time delays: Experimental results," IEEE Microw. Guided Wave Lett., vol. 6, no. 8, p. 283, Aug. 1996.

[9] G. Appenzeller, N. McKeown, J. Sommers, and P. Barford, "Recent results on sizing router buffers," in Proc. Network Systems Design Conf., San Diego, CA, Oct. 18-20, 2004, p. 13.

[10] "Cisco CRS-1 8-slot line-card chassis route processor," CISCO Data Sheet, 2005. p. 8.

[11] T. P. Haraszti, CMOS Memory Circuits. Norwell, MA: Kluwer, 2000.

[12] C. Liu, Z. Dutton, C. Behroozi, and L. V. Hau, "Observation of coherent optical information storage in an atomic medium using halted light pulses," Nature, vol. 409, no. 6819, pp. 490-493, Jan. 25, 2001.

[13] L. V. Hau, S. E. Harris, Z. Dutton, and C. H. Behroozi, "Light speed reduction to 17 meters per second in an ultracold atomic gas," Nature, vol. 397, no. 6720, pp. 594-598, Feb. 18, 1999

[14] P. C. Ku, F. Sedgwich, C. Chang-Hasnain, P. Palinginis, T. Li, H. Wang, S. W. Chang, and S. L. Chuang, "Slow light in semiconductor quantum wells," Opt. Lett., vol. 29, no. 19, pp. 2291-2293, 2004.
[15] A. Turukhin, V. S. Sudarshanam, M. S. Shahriar, J. A. Musser, and P. R. Hemmer, "First observation of ultraslow group velocity of light in a solid," in Tech. Dig., Quantum Electronics Laser Science Conf. (QELS), Baltimore, MD, May 6-11, 2001, pp. 6-7.

[16] A. Yariv, Y. Xu, R. K. Lee, and A. Scherer, "Coupled-resonator optical waveguide: A proposal and analysis," Opt. Lett., vol. 24, no. 11, pp. 711-713, 1999.

[17] M. Soljacic, S. Johnson, S. Fan, M. Ibanescu, E. Ippen, and J. D. Joannopoulos, "Photonic-crystal slow-light enhancement of nonlinear phase sensitivity," J. Opt. Soc. Amer. B, vol. 19, no. 9, pp. 2052-2059, 2002.

[18] D. K. Hunter, M. C. Chia, and I. Andonovic, "Buffering in optical packet switches," J. Lightw. Technol., vol. 16, no. 12, pp. 2081-2094, Dec. 1998.

[19] C. J. Chang-Hasnain, P. C. Ku, J. Kim, and S. L. Chuang, "Variable optical buffer using slow light in semiconductor nanostructures," Proc. IEEE, vol. 91, no. 11, pp. 1884-1897, 2003.

[20] P. C. Ku, C. J. Chang-Hasnain, and S. L. Chuang, "A proposal of variable semiconductor all-optical buffer," Electron. Lett., vol. 38, no. 24, pp. 1581-1583, 2002.

[21] R. S. Tucker, P.-C. Ku, and C. J. Chang-Hasnain, "Delay-bandwidth product and storage density in slow-light optical buffers," Electron. Lett., vol. 41, no. 4, pp. 61-62, Feb. 2005.

[22] P. C. Ku, F. Sedgwick, C. J. Chang-Hasnain, and R. S. Tucker, "Link performance of all-optical buffers using slow light," in Proc. Optical Fiber Communication Conf. (OFC), Anaheim, CA, Mar. 2005, p. 2.

[23] J. R. Pierce, Travelling-Wave Tubes. Princeton, NJ: Van Nostrand, 1950.

[24] G. Lenz, B. J. Eggleton, C. K. Madsen, and R. E. Slusher, "Optical delay lines based on optical filters," IEEE J. Quantum Electron., vol. 37, no. 4, pp. 525-532, Apr. 2001.

[25] U. Troppenez, M. Hamacher, D. G. Rabus, and H. Heidrich, "All-active InGaAsP/InP ring cavities for widespread functionalities in the wavelength domain," in Proc. Indium Phosphide Related Materials Conf. (IPRM), Stockholm, Sweden, May 2002, pp. 475-478.

[26] A. Melloni, F. Morichetti, and M. Martinelli, "Linear and nonlinear pulse propagation in coupled resonator slow-wave optical structures," Opt. Quantum Electron., vol. 35, no. 4, pp. 365-379, 2003.

[27] P. Ku, C. Chang-Hasnain, J. Kim, and S. Chuang, "Semiconductor all-optical buffers using quantum dots in resonator structures," in Tech. Dig., Optical Fiber Communications Conf., Atlanta, GA, Mar. 23-28, 2003, vol. 1M, pp. 76-78.

[28] Y. Xu, A. Yariv, C. Gunn, and R. K. Lee, "Tunable group velocity reduction in coupled-resonator optical waveguide (CROW)," in Quantum Electronics Laser Science Conf. (QELS), Tech. Dig., San Francisco, CA, May 2000, pp. 93-94.

[29] M. F. Yanik and S. Fan, "Stopping light all optically," Phys. Rev. Lett., vol. 92, no. 8, pp. 083901-1-083901-4, Feb. 27, 2004.

[30] J. S. Foresi, P. R. Villeneuve, J. Ferrera, E. R. Thoen, G. Steinmeyer, S. Fan, J. D. Joannopoulos, L. C. Kimerling, H. I. Smith, and E. P. Ippen, "Photonic-bandgap microcavities in optical waveguides," Nature, vol. 390, no. 6656, pp. 143-145, 1997.

[31] S. Mookherjea and A. Yariv, "Coupled resonator optical waveguides," IEEE J. Sel. Topics Quantum Electron., vol. 8, no. 3, pp. 448-456, May-Jun. 2002.

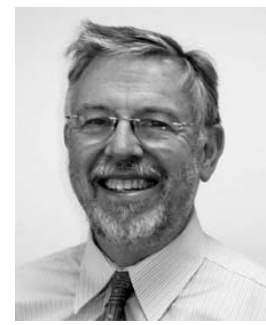

Rodney S. Tucker (S'72-M'78-SM'85-F'90) received the B.E. and $\mathrm{Ph} . \mathrm{D}$. degrees from the University of Melbourne, Melbourne, Vic., Australia, in 1969 and 1975 , respectively.

$\mathrm{He}$ is a Laureate Professor at the University of Melbourne. He is the Research Director of the Australian Research Council Special Research Centre for Ultra-Broadband Information Networks in the University of Melbourne's Department of Electrical and Electronic Engineering. He has held positions at the University of Queensland, the University of California, Berkeley, Cornell University, Plessey Research, AT\&T Bell Laboratories, Hewlett Packard Laboratories, and Agilent Technologies.

Prof. Tucker is a fellow of the Australian Academy of Science and a fellow of the Australian Academy of Technological Sciences and Engineering. In 1997, he was awarded the Australia Prize for his contributions to telecommunications. 


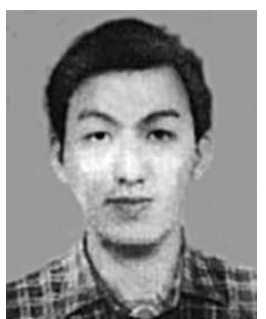

Pei-Cheng Ku received the B.S. degree from the National Taiwan University, Taipei, Taiwan R.O.C., in 1995, and the Ph.D. degree from the University of California, Berkeley, in 2003, both in electrical engineering. He was a recipient of the Berkeley Fellowship during his Ph.D. study.

From 2003 to 2004, he was a Postdoctoral Researcher in the Center for Optoelectronic Nanostructured Semiconductor Technologies (CONSRT) at Berkeley, where his research focused on slow light and nanophotonic materials. From 2004 to 2005, he was a Senior Process Engineer with Intel Corporation, where his research focused on the phase change memory. He is currently an Assistant Professor in the Department of Electrical Engineering and Computer Science, University of Michigan, Ann Arbor. His research interests include nanophotonics, nanofabrication, and biophotonics.

Dr. Ku has been an honorary member of Phi-Tau-Phi since 1995. He received the Ross Tucker Memorial Award in 2004.

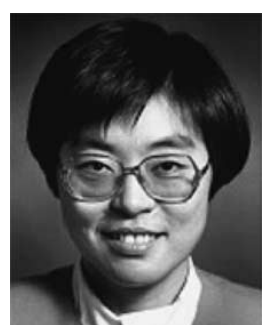

Constance J. Chang-Hasnain (M'88-SM'92F'98) received the B.S. degree from the University of California, Davis, in 1982 and the M.S. and Ph.D. degrees from the University of California, Berkeley, in 1984, and 1987, respectively, all in electrical engineering.

She was a member of Technical Staff at Bellcore from 1987 to 1992. From April 1992 to December 1995, she was an Associate Professor of Electrical Engineering at Stanford University, Stanford, CA. Since January 1996, she has been a Professor in the Department of Electrical Engineering, University of California, Berkeley. Since 2004, she has been the Director of the Center for Optoelectronic Nanostructured Semiconductor Technologies (CONSRT). Her research interests are in nanostructured materials, nano- and microoptoelectronic devices, and their applications. She coauthored 283 research papers in technical journals and conferences, and six book chapters. She also has 26 patents granted.

Prof. Chang-Hasnain is a fellow of the OSA and IEE. She was elected as an honorary member of A. F. Ioffe Institute in 2005. She was named a Presidential Faculty Fellow, a National Young Investigator, a Packard Fellow, a Sloan Research Fellow, and Outstanding Young Electrical Engineer of the Year by Eta Kappa Nu. She received the 1994 IEEE LEOS Distinguished Lecturer Award, the 2000 Curtis W. McGraw Research Award from the American Society of Engineering Education, the 2003 IEEE William Streifer Scientific Achievement Award, and the 2005 Gilbreth Lecturer Award from the National Academy of Engineering. She is an Associate Editor for IEEE JournAL OF LightwaVE TECHNOLOGIES. 